\title{
NUMERICAL APPROXIMATION OF THE SPECTRUM OF THE CURL OPERATOR
}

\author{
RODOLFO RODRÍGUEZ AND PABLO VENEGAS
}

\begin{abstract}
The aim of this paper is to study the numerical approximation of the eigenvalue problem for the curl operator. The three-dimensional divergencefree eigensolutions of this problem are examples of the so-called Beltrami fields or linear force-free fields, which arise in various physics areas such as solar physics, plasma physics, and fluid mechanics. The present analysis is restricted to bounded simply-connected domains. A finite element discretization of a convenient weak formulation of the spectral problem is proposed and analyzed. Optimal-order spectral convergence is proved, as well as absence of spurious modes. The results of some numerical tests are also reported.
\end{abstract}

\section{INTRODUCTION}

The aim of this paper is to study the numerical approximation of the spectrum of the curl operator. More precisely, we focus on the following eigenvalue problem: find $\lambda \in \mathbb{C}$ and $\boldsymbol{u} \neq \mathbf{0}$ such that

$$
\begin{aligned}
\operatorname{curl} \boldsymbol{u}=\lambda \boldsymbol{u} & \text { in } \Omega, \\
\operatorname{div} \boldsymbol{u}=0 & \text { in } \Omega, \\
\boldsymbol{u} \cdot \boldsymbol{n}=0 & \text { on } \Gamma,
\end{aligned}
$$

where $\Omega$ is a bounded domain with boundary $\Gamma$ and outer unit normal vector $\boldsymbol{n}$. To analyze this problem, Yoshida and Giga studied in [25] the spectral properties of the curl in various functions spaces. In particular, they show that if $\Omega$ is multiplyconnected, then the problem above has a nontrivial solution for any complex $\lambda$. Because of this, we restrict our analysis to simply-connected domains.

The spectral problem for the curl operator has a longstanding tradition in mathematical physics. A large measure of the credit goes to Beltrami [2], who seems to be the first who considered this problem in the context of fluid dynamics. This is the reason why the corresponding eigenfunctions are called Beltrami fields (also Trkalian fields [22]; we refer to [15] for a brief survey on the history of this subject). Such fields are useful in solar physics for testing theories on flares and coronal heating, in fluid mechanics for the study of the static equilibrium of smectic liquid crystals, and in superconducting materials, just to name a few; even particle movement in tornadoes and waterspouts can be approximated by Beltrami fields.

On the other hand, the eigenfunctions of this spectral problem are particular cases of the so-called force-free fields. These are vector fields which satisfy the first equation of the eigenvalue problem above, with $\lambda$ not necessarily a constant but a

Received by the editor July 21, 2011.

1991 Mathematics Subject Classification. Primary 65N25, 65N30; Secondary 76M10, 78M10.

Key words and phrases. Eigenvalue problems, finite elements, spectrum of the curl operator, Beltrami fields, linear force-free fields. 
scalar function. The name arises from magnetohydrodynamics, since a magnetic field $\boldsymbol{H}$ satisfying such an equation, induces a vanishing Lorentz force: $\boldsymbol{F}:=\boldsymbol{J} \times$ $\boldsymbol{B}=\operatorname{curl} \boldsymbol{H} \times(\mu \boldsymbol{H})=\mathbf{0}$. In [23], Woltjer showed that the lowest state of magnetic energy density within a closed system is attained when $\lambda$ is spatially constant. In such a case $\boldsymbol{H}$ is called a linear force-free field and its determination is naturally related with the spectral problem for the curl operator. The eigenfunctions of this problem are also known as free-decay fields or Taylor fields and play an important role, for instance, in the study of turbulence in plasma physics [21].

The boundary condition $\boldsymbol{u} \cdot \boldsymbol{n}=0$ is the most natural one for a bounded domain and corresponds to a field confined within it. Analytical solutions of this problem are only known under particular symmetry assumptions. The first one was obtained by Chandrasekhar and Kendall for spherical domains in the context of astrophysical plasmas arising in modeling of the solar crown [11] (see also [10, 23, 24]). More recently, Morse [19] studied the problem on cylindrical bounded domains.

On general domains, Boulmezaud, Maday, and Amari studied in [7] different boundary value problems whose solutions are linear force-free fields and they prove existence, uniqueness, and regularity of the solution. Based on the analysis of that paper, Boulmezaud and Amari proposed and analyzed finite element discretizations for numerically solving various linear [5] and non-linear [6] force-free field problems.

In this paper, we focus on the spectral problem above. We consider a weak formulation, which, after discretization, leads to a well-posed generalized eigenvalue problem. We propose a method for its numerical solution based on Nédélec finite elements of arbitrary order [20]. By using the spectral theory for non-compact operators from $[12,13]$, we prove spectral convergence and establish optimal-order error estimates. We also prove that the method is free of spurious modes. However, although the method always allows approximating the eigenvalues, in case of different eigenvalues with the same absolute value, it is not able to distinguish between the respective eigenfunctions. Because of this, we propose and analyze in an appendix an alternative numerical method based on the finite element discretization of other weak formulation. Although this approach leads to a degenerate generalized eigenvalue problem, we prove its well-posedness and spectral convergence.

The outline of the paper is as follows. In Section 2, we introduce some function spaces that will be used in the sequel. Then, in Section 3 , we give a first weak formulation and prove that it is equivalent to the spectral problem for a self-adjoint compact operator. This allows us to obtain a thorough characterization of the solutions of the eigenproblem. However, a direct discretization of the given weak formulation would lead to a degenerate generalized eigenvalue problem. To avoid this, we propose a second weak formulation more amenable for numerical purposes. In Section 4, we introduce a finite element discretization. We prove optimal-order spectral convergence and absence of spurious modes. We describe how to efficiently implement this methods in Section 5. In Section 6, we report the results of a couple of numerical tests, which allow us to check the theoretical results and to assess the performance of the method. Finally, in an appendix, we introduce and analyze a finite element discretization of an alternative weak form.

\section{Preliminaries}

Let $\Omega \in \mathbb{R}^{3}$ be a bounded simply-connected domain with a Lipschitz continuous boundary $\Gamma$. We assume that $\Omega$ is bounded and either $\Gamma$ is smooth or $\Omega$ is a 
polyhedron. Let $\Gamma_{0}, \ldots, \Gamma_{I}$ be the connected components of $\Gamma$, with $\Gamma_{0}$ being the boundary of the only unbounded connected component of $\mathbb{R}^{3} / \bar{\Omega}$.

We consider the space $\mathrm{L}^{2}(\Omega)$ with its corresponding norm $\|\cdot\|_{0, \Omega}$; for convenience, we denote $\|\cdot\|_{0, \Omega}$ the norm of $\mathrm{L}^{2}(\Omega)^{3}$, too. As usual, for all $s>0$, we consider the Hilbertian Sobolev space $\mathrm{H}^{s}(\Omega)$ with norm $\|\cdot\|_{s, \Omega}$; we also denote by $\|\cdot\|_{s, \Omega}$ the norm of the space $\mathrm{H}^{s}(\Omega)^{3}$.

Let $\mathcal{D}(\Omega)$ be the space of infinitely differentiable functions with compact support in $\Omega$ and $\mathcal{D}(\bar{\Omega}):=\left\{\left.\phi\right|_{\Omega}: \phi \in \mathcal{D}\left(\mathbb{R}^{3}\right)\right\}$.

Let $\mathrm{H}^{1 / 2}(\Gamma)$ be the space of traces on $\Gamma$ of functions in $\mathrm{H}^{1}(\Omega)$, with dual space $\mathrm{H}^{-1 / 2}(\Gamma)$ and dual pairing $\langle\cdot, \cdot\rangle_{\Gamma}$.

Throughout the paper, we will use the Hilbert spaces

$$
\begin{aligned}
\mathrm{H}(\operatorname{curl} ; \Omega) & :=\left\{\boldsymbol{v} \in \mathrm{L}^{2}(\Omega)^{3}: \operatorname{curl} \boldsymbol{v} \in \mathrm{L}^{2}(\Omega)^{3}\right\}, \\
\mathrm{H}(\operatorname{div} ; \Omega) & :=\left\{\boldsymbol{v} \in \mathrm{L}^{2}(\Omega)^{3}: \operatorname{div} \boldsymbol{v} \in \mathrm{L}^{2}(\Omega)\right\},
\end{aligned}
$$

with their respective norms defined by $\|\boldsymbol{v}\|_{\text {curl, }, \Omega}^{2}:=\|\boldsymbol{v}\|_{0, \Omega}^{2}+\|\operatorname{curl} \boldsymbol{v}\|_{0, \Omega}^{2}$ and $\|\boldsymbol{v}\|_{\operatorname{div}, \Omega}^{2}:=\|\boldsymbol{v}\|_{0, \Omega}^{2}+\|\operatorname{div} \boldsymbol{v}\|_{0, \Omega}^{2}$, and the followings closed subspaces:

$$
\begin{aligned}
\mathrm{H}_{0}(\operatorname{div} ; \Omega) & :=\{\boldsymbol{v} \in \mathrm{H}(\operatorname{div} ; \Omega): \boldsymbol{v} \cdot \boldsymbol{n}=0 \text { on } \Gamma\}, \\
\mathrm{H}_{0}(\operatorname{curl} ; \Omega) & :=\{\boldsymbol{v} \in \mathrm{H}(\operatorname{curl} ; \Omega): \boldsymbol{v} \times \boldsymbol{n}=\mathbf{0} \text { on } \Gamma\}, \\
\mathrm{H}\left(\operatorname{div}^{0} ; \Omega\right) & :=\{\boldsymbol{v} \in \mathrm{H}(\operatorname{div} ; \Omega): \operatorname{div} \boldsymbol{v}=0 \text { in } \Omega\}, \\
\mathrm{H}\left(\operatorname{curl}^{0} ; \Omega\right) & :=\{\boldsymbol{v} \in \mathrm{H}(\operatorname{curl} ; \Omega): \operatorname{curl} \boldsymbol{v}=\mathbf{0} \text { in } \Omega\}, \\
\mathrm{H}_{0}\left(\operatorname{div}^{0} ; \Omega\right) & :=\mathrm{H}_{0}(\operatorname{div} ; \Omega) \cap \mathrm{H}\left(\operatorname{div}^{0} ; \Omega\right), \\
\mathrm{H}_{0}\left(\operatorname{curl}^{0} ; \Omega\right) & :=\mathrm{H}_{0}(\operatorname{curl} ; \Omega) \cap \mathrm{H}\left(\operatorname{curl}^{0} ; \Omega\right) .
\end{aligned}
$$

Notice that the conditions $\boldsymbol{v} \cdot \boldsymbol{n}=0$ and $\boldsymbol{v} \times \boldsymbol{n}=\mathbf{0}$ on $\Gamma$, must be understood in the sense of $\mathrm{H}^{-1 / 2}(\Gamma)$.

The spaces $\mathrm{H}\left(\operatorname{curl}^{0} ; \Omega\right) \cap \mathrm{H}_{0}\left(\operatorname{div}^{0} ; \Omega\right)$ and $\mathrm{H}_{0}\left(\operatorname{curl}^{0} ; \Omega\right) \cap \mathrm{H}\left(\operatorname{div}^{0} ; \Omega\right)$ will also appear often in the sequel. Under the assumption of $\Omega$ being simply-connected the former is trivial (see, for instance, [14, Remark I.2.2]):

$$
\mathrm{H}\left(\operatorname{curl}^{0} ; \Omega\right) \cap \mathrm{H}_{0}\left(\operatorname{div}^{0} ; \Omega\right)=\{\mathbf{0}\} .
$$

This is not the case for the latter. The following characterization can be found in [1, Proposition 3.18].

Lemma 2.1. The dimension of $\mathrm{H}_{0}\left(\operatorname{curl}^{0} ; \Omega\right) \cap \mathrm{H}\left(\operatorname{div}^{0} ; \Omega\right)$ is equal to $I$. It is spanned by the functions $\nabla q_{i}, 1 \leq i \leq I$, where each $q_{i} \in \mathrm{H}^{1}(\Omega)$ is the unique solution of the problem

$$
\left\{\begin{array}{l}
-\Delta q_{i}=0 \quad \text { in } \Omega, \\
\left.q_{i}\right|_{\Gamma_{0}}=0 \text { and }\left.q_{i}\right|_{\Gamma_{k}}=\text { constant, } 1 \leq k \leq I, \\
\left\langle\partial_{n} q_{i}, 1\right\rangle_{\Gamma_{0}}=-1 \quad \text { and } \quad\left\langle\partial_{n} q_{i}, 1\right\rangle_{\Gamma_{k}}=\delta_{i k}, 1 \leq k \leq I .
\end{array}\right.
$$

Finally, we will also use the space

$$
\mathrm{H}^{s}(\operatorname{curl} ; \Omega):=\left\{\boldsymbol{v} \in \mathrm{H}^{s}(\Omega)^{3}: \operatorname{curl} \boldsymbol{v} \in \mathrm{H}^{s}(\Omega)^{3}\right\}, \quad s>0 .
$$

Let us recall that there exists $s>1 / 2$, only depending on the domain $\Omega$, such that the inclusions

$$
\mathrm{H}_{0}(\operatorname{curl} ; \Omega) \cap \mathrm{H}(\operatorname{div} ; \Omega) \hookrightarrow \mathrm{H}^{s}(\Omega)^{3}, \quad \mathrm{H}(\operatorname{curl} ; \Omega) \cap \mathrm{H}_{0}(\operatorname{div} ; \Omega) \hookrightarrow \mathrm{H}^{s}(\Omega)^{3}
$$


are continuous (see, for instance, [1], Proposition 3.7, if $\Omega$ is a polyhedron, and Theorems 2.9 and 2.12, if $\Gamma$ is smooth).

Throughout the paper, $C$ will denote a generic constant, not necessarily the same at each occurrence.

\section{SpeCtral Problem For the CURL OPERATOR}

We consider the following problem:

Problem 1. Find $\lambda \in \mathbb{C}$ and $\boldsymbol{u} \in \mathrm{H}(\operatorname{curl} ; \Omega), \boldsymbol{u} \neq \mathbf{0}$, such that

$$
\begin{aligned}
\operatorname{curl} \boldsymbol{u}=\lambda \boldsymbol{u} & \text { in } \Omega, \\
\operatorname{div} \boldsymbol{u}=0 & \text { in } \Omega, \\
\boldsymbol{u} \cdot \boldsymbol{n}=0 & \text { on } \Gamma .
\end{aligned}
$$

Notice that, for any solution of this problem, $\lambda \neq 0$. In fact, $\lambda=0$ would imply $\boldsymbol{u} \in \mathrm{H}\left(\operatorname{curl}^{0} ; \Omega\right) \cap \mathrm{H}_{0}\left(\operatorname{div}^{0} ; \Omega\right)$ and this space is trivial in our case.

The following is a mixed formulation of this problem.

Problem 2. Find $\lambda \in \mathbb{C}$ and $(\boldsymbol{u}, \varphi) \in \mathrm{H}(\operatorname{curl} ; \Omega) \times \mathrm{H}^{1}(\Omega) / \mathbb{C},(\boldsymbol{u}, \varphi) \neq \mathbf{0}$, such that

$$
\begin{aligned}
& \int_{\Omega} \operatorname{curl} \boldsymbol{u} \cdot \operatorname{curl} \overline{\boldsymbol{v}}+\int_{\Omega} \nabla \varphi \cdot \overline{\boldsymbol{v}}=\lambda \int_{\Omega} \boldsymbol{u} \cdot \operatorname{curl} \overline{\boldsymbol{v}} \quad \forall \boldsymbol{v} \in \mathrm{H}(\operatorname{curl} ; \Omega), \\
& \int_{\Omega} \boldsymbol{u} \cdot \nabla \bar{\psi}=0 \quad \forall \psi \in \mathrm{H}^{1}(\Omega) / \mathbb{C} .
\end{aligned}
$$

In order to establish the equivalence of these two problems, first note that the last two equations of the former are equivalent to the last equation of the latter. Hence, it is clear that if $(\lambda, \boldsymbol{u})$ is a solution of Problem 1, then, $(\lambda, \boldsymbol{u}, 0)$ solves Problem 2. Conversely, if $(\lambda, \boldsymbol{u}, \varphi)$ is a solution of Problem 2, by taking $\boldsymbol{v}=\nabla \varphi$ in its first equation, we conclude that $\varphi=0$. Therefore, we only need to prove that $\operatorname{curl} \boldsymbol{u}=\lambda \boldsymbol{u}$ in $\Omega$.

With this end, we test the first equation of Problem 2 with $\boldsymbol{v} \in \mathcal{D}(\Omega)^{3}$ and integrate by parts to conclude that

$$
\operatorname{curl}(\operatorname{curl} \boldsymbol{u}-\lambda \boldsymbol{u})=\mathbf{0} \text { in } \Omega .
$$

Then, taking $\boldsymbol{v} \in \mathrm{H}^{1}(\Omega)^{3}$, by integration by parts it follows that

$$
(\operatorname{curl} \boldsymbol{u}-\lambda \boldsymbol{u}) \times \boldsymbol{n}=\mathbf{0} \quad \text { on } \Gamma .
$$

Thus, we have that $\operatorname{curl} \boldsymbol{u}-\lambda \boldsymbol{u} \in \mathrm{H}_{0}\left(\operatorname{curl}^{0} ; \Omega\right) \cap \mathrm{H}\left(\operatorname{div}^{0} ; \Omega\right)$. Therefore, from Lemma 2.1, it follows that there exists $q \in \mathrm{H}^{1}(\Omega)$, with $\left.q\right|_{\Gamma_{0}}=0$ and $\left.q\right|_{\Gamma_{k}}=C_{k}$ (constant), $1 \leq k \leq I$, such that $\operatorname{curl} \boldsymbol{u}-\lambda \boldsymbol{u}=\nabla q$. Then, integrating by parts we have

$$
\begin{aligned}
\|\nabla q\|_{0, \Omega}^{2} & =\int_{\Omega}(\operatorname{curl} \boldsymbol{u}-\lambda \boldsymbol{u}) \cdot \nabla \bar{q}=\int_{\Omega} \operatorname{curl} \boldsymbol{u} \cdot \nabla \bar{q} \\
& =\langle\operatorname{curl} \boldsymbol{u} \cdot \boldsymbol{n}, q\rangle_{\Gamma}=\sum_{k=1}^{I} C_{k}\langle\operatorname{curl} \boldsymbol{u} \cdot \boldsymbol{n}, 1\rangle_{\Gamma_{k}}=0 .
\end{aligned}
$$

Whence, $\nabla q=0$ and, consequently, $\operatorname{curl} \boldsymbol{u}=\lambda \boldsymbol{u}$ in $\Omega$. Thus we conclude the following equivalence result. 
Proposition 3.1. If $(\lambda, \boldsymbol{u})$ is a solution of Problem 1, then $(\lambda, \boldsymbol{u}, 0)$ is a solution of Problem 2. Conversely, if $(\lambda, \boldsymbol{u}, \varphi)$ is a solution of Problem 2, then $\varphi=0$ and $(\lambda, \boldsymbol{u})$ is a solution of Problem 1.

For the analysis of Problem 2, we consider the following solution operator:

$$
\begin{aligned}
S: \mathrm{H}_{0}\left(\operatorname{div}^{0} ; \Omega\right) & \longrightarrow \mathrm{H}_{0}\left(\operatorname{div}^{0} ; \Omega\right), \\
\boldsymbol{f} & \longmapsto S \boldsymbol{f}:=\boldsymbol{w},
\end{aligned}
$$

with $\boldsymbol{w} \in \mathrm{H}(\operatorname{curl} ; \Omega)$ such that there exists $\xi \in \mathrm{H}^{1}(\Omega) / \mathbb{C}$ satisfying

$$
\begin{aligned}
& \int_{\Omega} \operatorname{curl} \boldsymbol{w} \cdot \operatorname{curl} \overline{\boldsymbol{v}}+\int_{\Omega} \nabla \xi \cdot \overline{\boldsymbol{v}}=\int_{\Omega} \boldsymbol{f} \cdot \operatorname{curl} \overline{\boldsymbol{v}} \quad \forall \boldsymbol{v} \in \mathrm{H}(\operatorname{curl} ; \Omega), \\
& \int_{\Omega} \boldsymbol{w} \cdot \nabla \bar{\psi}=0 \quad \forall \psi \in \mathrm{H}^{1}(\Omega) / \mathbb{C} .
\end{aligned}
$$

The Babuška-Brezzi conditions for this mixed problem are easy to check. In particular, the ellipticity in the kernel follows from the fact that, since $\Omega$ is simplyconnected, $\|\boldsymbol{v}\|_{\text {curl }, \Omega} \leq C\|\operatorname{curl} \boldsymbol{v}\|_{0, \Omega}$ for all $\boldsymbol{v} \in \mathrm{H}(\operatorname{curl} ; \Omega) \cap \mathrm{H}_{0}\left(\operatorname{div}^{0} ; \Omega\right)$; see [1, Corollary 3.16]). Consequently, (3.1)-(3.2) has a unique solution $(\boldsymbol{w}, \xi)$, which satisfies $\xi=0$ and $\|\boldsymbol{w}\|_{\operatorname{curl}, \Omega} \leq C\|f\|_{0, \Omega}$. Moreover, (3.2) shows that $\boldsymbol{w} \in \mathrm{H}_{0}\left(\operatorname{div}^{0} ; \Omega\right)$. Hence, the operator $S$ is well-defined and continuous.

Clearly, $S \boldsymbol{u}=\mu \boldsymbol{u}$, with $\mu \neq 0$, if an only if $(\lambda, \boldsymbol{u}, 0)$ is a solution of Problem 2, with $\lambda=\frac{1}{\mu}$. Thus, we focus on characterizing the spectrum of $S$.

We note that $S\left(\mathrm{H}_{0}\left(\operatorname{div}^{0} ; \Omega\right)\right) \subset \mathrm{H}(\operatorname{curl} ; \Omega) \cap \mathrm{H}_{0}\left(\operatorname{div}^{0} ; \Omega\right)$. Since, according to (2.1), there exists $s>1 / 2$ such that

$$
\mathrm{H}(\mathbf{c u r l} ; \Omega) \cap \mathrm{H}_{0}\left(\operatorname{div}^{0} ; \Omega\right) \hookrightarrow \mathrm{H}^{s}(\Omega)^{3} \cap \mathrm{H}_{0}\left(\operatorname{div}^{0} ; \Omega\right) \hookrightarrow \mathrm{H}_{0}\left(\operatorname{div}^{0} ; \Omega\right),
$$

the first inclusion being continuous and the second one compact (cf. [14, Theorem I.1.3]), we conclude that $S$ is compact.

Moreover, from (3.1), by proceeding as for Problem 2 we obtain

$$
\operatorname{curl}(\operatorname{curl} \boldsymbol{w}-\boldsymbol{f})=\mathbf{0} \quad \text { in } \Omega \quad \text { and } \quad(\operatorname{curl} \boldsymbol{w}-\boldsymbol{f}) \times \boldsymbol{n}=\mathbf{0} \quad \text { on } \Gamma .
$$

Hence, using Lemma 2.1 again, it is straightforward to show that $\operatorname{curl} \boldsymbol{w}=\boldsymbol{f}$ in $\Omega$. Thus $\boldsymbol{w}$ belongs to the space

$$
\mathcal{Z}:=\{\boldsymbol{v} \in \mathrm{H}(\operatorname{curl} ; \Omega): \operatorname{curl} \boldsymbol{v} \cdot \boldsymbol{n}=0 \text { on } \Gamma\} .
$$

We summarize these results in the following lemma.

Lemma 3.2. Operator $S$ is compact. Moreover, $S\left(\mathrm{H}_{0}\left(\operatorname{div}^{0} ; \Omega\right)\right) \subset \mathcal{Z}$ and, for all $\boldsymbol{f} \in \mathrm{H}_{0}\left(\operatorname{div}^{0} ; \Omega\right)$, if $\boldsymbol{w}=S \boldsymbol{f}$, then $\operatorname{curl} \boldsymbol{w}=\boldsymbol{f}$ in $\Omega$.

Next step is to establish some properties of the space $\mathcal{Z}$ that will be used in the sequel. The first one is the following result, which has been proved in [25, Theorem 1] in a more general setting (see also [16, Proposition 2.3]). We include here an elementary proof, for completeness.

Proposition 3.3. For all $\boldsymbol{y}, \boldsymbol{z} \in \mathcal{Z}$

$$
\int_{\Omega}(\operatorname{curl} \boldsymbol{y} \cdot \bar{z}-\boldsymbol{y} \cdot \operatorname{curl} \bar{z})=0
$$


Proof. Let $\boldsymbol{y} \in \mathcal{Z}$. Then $\operatorname{curl} \boldsymbol{y} \in \mathrm{H}_{0}\left(\operatorname{div}^{0} ; \Omega\right)$. Since $\Omega$ is simply-connected, we know (cf. [14, Theorem I.3.6]) that there exists a unique $\boldsymbol{\Phi} \in \mathrm{H}_{0}(\operatorname{curl} ; \Omega) \cap$ $\mathrm{H}\left(\operatorname{div}^{0} ; \Omega\right)$, such that

$$
\operatorname{curl} y=\operatorname{curl} \Phi \quad \text { in } \Omega
$$

and, consequently, there exists a unique $\psi \in \mathrm{H}^{1}(\Omega) / \mathbb{C}$, such that

$$
\boldsymbol{y}=\nabla \psi+\boldsymbol{\Phi} \quad \text { in } \Omega \text {. }
$$

Then, for $\boldsymbol{z} \in \mathcal{Z}$, we have

$$
\int_{\Omega}(\operatorname{curl} \boldsymbol{y} \cdot \overline{\boldsymbol{z}}-\boldsymbol{y} \cdot \operatorname{curl} \overline{\boldsymbol{z}})=\int_{\Omega}(\operatorname{curl} \Phi \cdot \overline{\boldsymbol{z}}-\mathbf{\Phi} \cdot \operatorname{curl} \overline{\boldsymbol{z}})-\int_{\Omega} \nabla \psi \cdot \operatorname{curl} \overline{\boldsymbol{z}} .
$$

Now, since for all $\boldsymbol{v} \in \mathrm{H}^{1}(\Omega)^{3}$

$$
\int_{\Omega}(\operatorname{curl} \boldsymbol{\Phi} \cdot \overline{\boldsymbol{v}}-\boldsymbol{\Phi} \cdot \operatorname{curl} \overline{\boldsymbol{v}})=\langle\boldsymbol{\Phi} \times \boldsymbol{n}, \boldsymbol{v}\rangle_{\Gamma}=0
$$

and $\mathrm{H}^{1}(\Omega)^{3} \hookrightarrow \mathrm{H}(\mathbf{c u r l} ; \Omega)$ densely, we obtain

$$
\int_{\Omega}(\operatorname{curl} \Phi \cdot \bar{z}-\Phi \cdot \operatorname{curl} \bar{z})=0 .
$$

On the other hand, using integration by parts we have that

$$
\int_{\Omega} \nabla \psi \cdot \operatorname{curl} \overline{\boldsymbol{z}}=\langle\operatorname{curl} \boldsymbol{z} \cdot \boldsymbol{n}, \psi\rangle_{\Gamma}=0 .
$$

Thus, we conclude the proof.

A first consequence of the above proposition is the following density result for the smooth functions of $\mathcal{Z}$.

Proposition 3.4. Subspace $\mathcal{D}(\bar{\Omega})^{3} \cap \mathcal{Z}$ is dense in $\mathcal{Z}$.

Proof. The proof is based on a classical property (see, for instance, [14, Section I, (2.14)]), which in our case reads as follows: $\mathcal{D}(\bar{\Omega})^{3} \cap \mathcal{Z}$ is dense in $\mathcal{Z}$ if and only if every element of $\mathcal{Z}^{\prime}$ that vanish on $\mathcal{D}(\bar{\Omega})^{3} \cap \mathcal{Z}$ also vanishes on $\mathcal{Z}$.

Let $L \in \mathcal{Z}^{\prime}$. Since $\mathcal{Z}$ is a Hilbert space, there exists $\boldsymbol{l} \in \mathcal{Z}$ such that

$$
\langle L, \boldsymbol{v}\rangle=\int_{\Omega}(\boldsymbol{l} \cdot \overline{\boldsymbol{v}}+\tilde{\boldsymbol{l}} \cdot \operatorname{curl} \overline{\boldsymbol{v}}) \quad \forall \boldsymbol{v} \in \mathcal{Z},
$$

where $\langle\cdot, \cdot\rangle$ denote the duality pairing between $\mathcal{Z}^{\prime}$ and $\mathcal{Z}$ and $\tilde{\boldsymbol{l}}:=\operatorname{curl} \boldsymbol{l}$. Now, assume that $L$ vanishes on $\mathcal{D}(\bar{\Omega})^{3} \cap \mathcal{Z}$, namely,

$$
\langle L, \boldsymbol{v}\rangle=\int_{\Omega}(\boldsymbol{l} \cdot \overline{\boldsymbol{v}}+\tilde{\boldsymbol{l}} \cdot \operatorname{curl} \overline{\boldsymbol{v}})=0 \quad \forall \boldsymbol{v} \in \mathcal{D}(\bar{\Omega})^{3} \cap \mathcal{Z} .
$$

We need to prove that $L$ vanishes on $\mathcal{Z}$, too. With this end, note that since $\mathcal{D}(\Omega)^{3} \subset \mathcal{D}(\bar{\Omega})^{3} \cap \mathcal{Z}$, it follows that

$$
\int_{\Omega} \boldsymbol{l} \cdot \overline{\boldsymbol{v}}+\int_{\Omega} \tilde{\boldsymbol{l}} \cdot \operatorname{curl} \overline{\boldsymbol{v}}=0 \quad \forall \boldsymbol{v} \in \mathcal{D}(\Omega)^{3}
$$

and, hence, $\boldsymbol{l}=-\operatorname{curl} \tilde{\boldsymbol{l}}$. On the other hand, given that $\nabla(\mathcal{D}(\bar{\Omega})) \subset \mathcal{D}(\bar{\Omega})^{3} \cap \mathcal{Z}$ too, we have

$$
\int_{\Omega} \boldsymbol{l} \cdot \nabla \bar{\psi}=0 \quad \forall \psi \in \mathcal{D}(\bar{\Omega})
$$


Then, $\operatorname{curl} \tilde{\boldsymbol{l}}=-\boldsymbol{l} \in \mathrm{H}_{0}\left(\operatorname{div}^{0} ; \Omega\right)$, so that $\tilde{\boldsymbol{l}} \in \mathcal{Z}$. Therefore, using Proposition 3.3 we obtain

$$
\langle L, \boldsymbol{v}\rangle=\int_{\Omega}(\boldsymbol{l} \cdot \overline{\boldsymbol{v}}+\tilde{\boldsymbol{l}} \cdot \operatorname{curl} \overline{\boldsymbol{v}})=\int_{\Omega}(-\operatorname{curl} \tilde{\boldsymbol{l}} \cdot \overline{\boldsymbol{v}}+\tilde{\boldsymbol{l}} \cdot \operatorname{curl} \overline{\boldsymbol{v}})=0 \quad \forall \boldsymbol{v} \in \mathcal{Z} .
$$

This proves the claimed density.

Another consequence of Proposition 3.3 is the self-adjointness of the operator $S$ which, together with its compactness, will allow us to obtain a thorough characterization of its spectrum.

Proposition 3.5. $S$ is self-adjoint.

Proof. Given $\boldsymbol{f}, \boldsymbol{g} \in \mathrm{H}_{0}\left(\operatorname{div}^{0} ; \Omega\right)$, let $\boldsymbol{w}:=S \boldsymbol{f}$ and $\boldsymbol{v}:=S \boldsymbol{g}$. From Lemma 3.2, $\operatorname{curl} \boldsymbol{w}=\boldsymbol{f}$ and $\operatorname{curl} \boldsymbol{v}=\boldsymbol{g}$ in $\Omega$. Hence, by using Proposition 3.3, we have that

$$
\int_{\Omega}(S \boldsymbol{f}) \cdot \overline{\boldsymbol{g}}=\int_{\Omega} \boldsymbol{w} \cdot \overline{\boldsymbol{g}}=\int_{\Omega} \boldsymbol{w} \cdot \operatorname{curl} \overline{\boldsymbol{v}}=\int_{\Omega} \operatorname{curl} \boldsymbol{w} \cdot \overline{\boldsymbol{v}}=\int_{\Omega} \boldsymbol{f} \cdot \overline{\boldsymbol{v}}=\int_{\Omega} \boldsymbol{f} \cdot(\overline{S \boldsymbol{g}})
$$

and we conclude the proof.

Now, we are in a position to establish a spectral characterization of $S$.

Lemma 3.6. The spectrum of $S$ is given by $\sigma(S)=\left\{\mu_{n}\right\}_{n \in \mathbb{N}} \cup\{0\}$, with $\left\{\mu_{n}\right\}$ being a sequence of non-vanishing finite-multiplicity eigenvalues which converge to zero. Moreover, $\mu=0$ is not an eigenvalue of $S$ and there exists a Hilbertian basis $\left\{\boldsymbol{u}_{n}\right\}_{n \in \mathbb{N}}$ of $\mathrm{H}_{0}\left(\operatorname{div}^{0} ; \Omega\right)$ of eigenfunctions of $S$; i.e., such that $S \boldsymbol{u}_{n}=\mu_{n} \boldsymbol{u}_{n}, n \in \mathbb{N}$.

Proof. The result is a consequence of the classical spectral characterization of compact self-adjoint operators. There only remains to prove that $\mu_{n} \neq 0 \forall n \in \mathbb{N}$. We proceed by contradiction. Assume $\mu_{n}=0$. Hence, $\int_{\Omega} \boldsymbol{u}_{n} \cdot \operatorname{curl} \boldsymbol{v}=0 \forall \boldsymbol{v} \in$ $\mathrm{H}(\operatorname{curl} ; \Omega)$. Then, $\boldsymbol{u}_{n} \in \mathrm{H}_{0}\left(\operatorname{curl}^{0} ; \Omega\right) \cap \mathrm{H}_{0}\left(\operatorname{div}^{0} ; \Omega\right)=\{\mathbf{0}\}$.

The above lemma and the relation between the spectrum of $S$ and Problem 2, yields a thorough characterization for the solutions of the latter and, consequently, for the solutions of Problem 1.

Theorem 3.7. Problem 1 has a denumerable set of solutions $\left(\lambda_{n}, \boldsymbol{u}_{n}\right), n \in \mathbb{N}$, and $\left\{\boldsymbol{u}_{n}\right\}_{n \in \mathbb{N}}$ is a Hilbertian basis of $\mathrm{H}_{0}\left(\operatorname{div}^{0} ; \Omega\right)$.

One possible way to approximate the solutions of Problem 1 is to consider an appropriate discretization of the variational form given in Problem 2. We propose and analyze in the appendix a finite element method based on this approach. However, it leads to a generalized eigenvalue problem involving two non-definite matrices. The numerical solution of such a generalized eigenvalue problem is significantly more complicated due to its degenerate character. In what follows we introduce an alternative formulation which overcomes this drawback and will lead, after discretization, to a well posed generalized eigenvalue problem.

Notice that, for $\lambda \neq 0$, Problem 1 is equivalent to the following one: find $\lambda \in \mathbb{C}$ and $\boldsymbol{u} \in \mathrm{H}(\operatorname{curl} ; \Omega), \boldsymbol{u} \neq \mathbf{0}$, such that

$$
\begin{aligned}
\operatorname{curl} \boldsymbol{u}=\lambda \boldsymbol{u} & \text { in } \Omega, \\
\operatorname{curl} \boldsymbol{u} \cdot \boldsymbol{n}=0 & \text { on } \Gamma .
\end{aligned}
$$


Clearly, the solution $\boldsymbol{u}$ of the above problem belongs to $\mathcal{Z}$ and satisfies

$$
\int_{\Omega} \operatorname{curl} \boldsymbol{u} \cdot \operatorname{curl} \overline{\boldsymbol{v}}=\lambda \int_{\Omega} \boldsymbol{u} \cdot \operatorname{curl} \overline{\boldsymbol{v}}=\lambda \int_{\Omega} \operatorname{curl} \boldsymbol{u} \cdot \overline{\boldsymbol{v}}=\lambda^{2} \int_{\Omega} \boldsymbol{u} \cdot \overline{\boldsymbol{v}} \quad \forall \boldsymbol{v} \in \mathcal{Z},
$$

where we have also used Proposition 3.3. Therefore, we are led to consider the following problem:

Problem 3. Find $\lambda \in \mathbb{C}$ and $\boldsymbol{u} \in \mathcal{Z}, \boldsymbol{u} \neq \mathbf{0}$, such that

$$
\int_{\Omega} \operatorname{curl} \boldsymbol{u} \cdot \operatorname{curl} \overline{\boldsymbol{v}}=\lambda^{2} \int_{\Omega} \boldsymbol{u} \cdot \overline{\boldsymbol{v}} \quad \forall \boldsymbol{v} \in \mathcal{Z} .
$$

We have just proved the following result.

Lemma 3.8. If $(\lambda, \boldsymbol{u})$ is a solution of Problem 1, then it is a solution of Problem 3.

The converse is partially true. To prove it, we consider the solution operator:

$$
\begin{aligned}
T: \mathrm{L}^{2}(\Omega)^{3} & \longrightarrow \mathrm{L}^{2}(\Omega)^{3}, \\
\boldsymbol{f} & \longmapsto T \boldsymbol{f}:=\boldsymbol{w},
\end{aligned}
$$

with $\boldsymbol{w} \in \mathcal{Z}$ such that

$$
\int_{\Omega} \operatorname{curl} w \cdot \operatorname{curl} \overline{\boldsymbol{v}}+\int_{\Omega} w \cdot \overline{\boldsymbol{v}}=\int_{\Omega} \boldsymbol{f} \cdot \overline{\boldsymbol{v}} \quad \forall \boldsymbol{v} \in \mathcal{Z} .
$$

The well-posedness of problem (3.3) is a direct consequence of Lax Milgram lemma, whence $T$ is well-defined and continuous. Note that $T \boldsymbol{u}=\mu \boldsymbol{u}$, with $\mu \neq 0$, if an only if $(\lambda, \boldsymbol{u})$ is a solution of Problem 3, with $\lambda^{2}+1=\frac{1}{\mu}$.

Clearly $\mu=1$ is an eigenvalue of $T$ (correspondingly, $\lambda=0$ is an eigenvalue of Problem 3) with associated eigenspace

$$
\mathcal{K}:=\{\boldsymbol{v} \in \mathcal{Z}: \operatorname{curl} \boldsymbol{v}=\mathbf{0} \quad \text { in } \Omega\}=\nabla\left(\mathrm{H}^{1}(\Omega)\right) .
$$

Since $T$ is clearly self-adjoint (cf. (3.3)), the orthogonal complement of $\mathcal{K}$,

$$
\mathcal{K}^{\perp_{\mathrm{L}^{2}(\Omega)^{3}}}=\mathrm{H}_{0}\left(\operatorname{div}^{0} ; \Omega\right)
$$

is an invariant subspace for $T$. Therefore,

$$
\widehat{T}:=\left.T\right|_{\mathrm{H}_{0}\left(\operatorname{div}^{0} ; \Omega\right)}: \mathrm{H}_{0}\left(\operatorname{div}^{0} ; \Omega\right) \longrightarrow \mathrm{H}_{0}\left(\operatorname{div}^{0} ; \Omega\right)
$$

is a well-defined bounded operator and $\sigma(T)=\sigma(\widehat{T}) \cup\{1\}$. Moreover, since $T$ takes values in the space $\mathcal{Z} \subset \mathrm{H}(\operatorname{curl} ; \Omega)$ and, by virtue of $(2.1), \mathrm{H}(\operatorname{curl} ; \Omega) \cap$ $\mathrm{H}_{0}\left(\operatorname{div}^{0} ; \Omega\right) \hookrightarrow \mathrm{H}_{0}\left(\operatorname{div}^{0} ; \Omega\right)$ compactly, we derive the compactness of $\widehat{T}$.

The following theorem shows how the eigenpairs of $T$, with $\mu \neq 1$, are related with the solution of Problem 1.

Theorem 3.9. The following properties hold true:

a) The spectrum of $T$ decomposes as follows:

$$
\sigma(T)=\{1\} \cup\left\{\mu_{n}\right\}_{n \in \mathbb{N}} \cup\{0\} .
$$

Moreover:

- $\mu=1$ is an eigenvalue of $T$ with infinite-dimensional eigenspace $\mathcal{K}$;

- $\left\{\mu_{n}\right\}_{n \in \mathbb{N}}$ is a sequence of finite-multiplicity eigenvalues $\mu_{n} \in(0,1)$, $n \in \mathbb{N}$, which converge to 0 ;

- $\mu=0$ is not an eigenvalue of $T$. 
b) If $\lambda$ in an eigenvalue of Problem 1 with eigenspace $\mathcal{E}$, then $\mu=\frac{1}{1+\lambda^{2}}$ is an eigenvalue of $T$ and $\mathcal{E}$ is an invariant subspace of $T$.

c) If $\mu \neq 1$ is an eigenvalue of $T$ with eigenspace $\mathcal{E}$, then there exists an eigenvalue $\lambda$ of Problem 1 such that $\mu=\frac{1}{1+\lambda^{2}}$ and $\mathcal{E}$ is an invariant subspace of Problem 1.

Proof. We have already proved that $\mu=1$ is an eigenvalue of $T$ with corresponding eigenspace $\mathcal{K}$ and that $\sigma(T)=\sigma(\widehat{T}) \cup\{1\}$. Thus, the spectral characterization of $T$ is a consequence of the compactness of $\widehat{T}$. On the other hand, $\mu=0$ is not an eigenvalue of $T$; in fact, if $T \boldsymbol{f}=\mathbf{0}$, then it follows from (3.3) that $\boldsymbol{f} \perp \mathcal{Z} \supset \mathcal{D}(\Omega)^{3}$ dense in $\mathrm{L}^{2}(\Omega)^{3}$, so that $\boldsymbol{f}=\mathbf{0}$. Moreover, for all the eigenvalues $\mu_{n} \neq 1$, it is also easy to show from $(3.3)$ that $\mu_{n} \in(0,1)$. Thus, we conclude (a).

In its turn, Lemma 3.8 and the arguments above lead to (b).

It remains to prove that all the eigenvalues of $\widehat{T}$ are of the form $\mu=\frac{1}{1+\lambda^{2}}$, with $\lambda$ being an eigenvalue of Problem 1. In fact, according to Theorem 3.7, the sequence of eigenfunctions of Problem 1 is a Hilbertian basis of $\mathrm{H}_{0}\left(\operatorname{div}^{0} ; \Omega\right)$. Since we have already proved in (b) that all of them are eigenfunctions of $\widehat{T}$, this operator cannot have an additional eigenpair; otherwise, since $T$ is self adjoint, the additional eigenfunction would have to be orthogonal to the whole Hilbertian basis, which cannot happen. Thus, we conclude (c).

As a consequence of this theorem and the relation between the eigenpairs of Problem 3 and those of the operator $T$, we obtain the following result.

Corollary 3.10. Let $\nu \neq 0$ be an eigenvalue of Problem 3 and $\mathcal{E}$ the corresponding eigenspace. Then, there exists an eigenvalue $\lambda$ of Problem 1 such that $\nu=\lambda^{2}$ and $\mathcal{E}$ is an invariant subspace of Problem 1.

Remark 3.11. Notice that the eigenfunctions of Problem 3 are not necessarily eigenfunctions of Problem 1. In fact, if $\lambda$ and $-\lambda$ were both eigenvalues of Problem 1 , then $\lambda^{2}$ would be an eigenvalue of Problem 3, with multiplicity equal to the sum of the multiplicities of $\lambda$ and $-\lambda$. However, an eigenfunction of Problem 3 corresponding to $\lambda^{2}$ would be a linear combination of the eigenfunctions of $\lambda$ and $-\lambda$, but not necessarily an eigenfunction itself. As will be shown in Section 6.1, this is something that always happens when the domain $\Omega$ is symmetric.

\section{Finite element approximation}

In this section, we introduce a Galerkin approximation of Problem 3 and prove some convergence results. From now on, we assume that $\Omega$ is a polyhedral domain and $\left\{\mathcal{T}_{h}\right\}_{h>0}$ is a regular family of partitions of $\bar{\Omega}$ in tetrahedra $T$, so that $\bar{\Omega}=\bigcup_{T \in \mathcal{T}_{h}} T$. We denote by $\mathcal{T}_{h}^{\Gamma}$ the corresponding triangulation induced on the boundary of $\Omega$, namely, $\mathcal{T}_{h}^{\Gamma}:=\left\{F\right.$ face of $\left.T \in \mathcal{T}_{h}: F \subset \Gamma\right\}$.

For any $T \in \mathcal{T}_{h}$, let $\mathcal{N}^{k}(T):=\mathbb{P}_{k-1}(T)^{3} \oplus\left\{\boldsymbol{p} \in \overline{\mathbb{P}}_{k}(T)^{3}: \boldsymbol{p}(\boldsymbol{x}) \cdot \boldsymbol{x}=0\right\}$, where $\mathbb{P}_{k}$ is the set of polynomials of degree not greater than $k$ and $\overline{\mathbb{P}}_{k}$ the subset of homogeneous polynomials of degree $k$.

The corresponding global space to approximate $\mathrm{H}(\mathbf{c u r l} ; \Omega)$ is the space of functions that are locally in $\mathcal{N}^{k}(T)$ and have continuous tangential components across the faces of the triangulation $\mathcal{T}_{h}$. This is the well-known Nédélec space:

$$
\left.\mathcal{N}_{h}:=\left\{\boldsymbol{v}_{h} \in \mathrm{H}(\operatorname{curl} ; \Omega):\left.\boldsymbol{v}_{h}\right|_{T} \in \mathcal{N}^{k}(T) \quad \forall T \in \mathcal{T}_{h}\right)\right\}
$$


(for further details see, for instance, [18, Section 5.5]). Whence, the natural approximation space for $\mathcal{Z}$ is

$$
\mathcal{Z}_{h}:=\mathcal{N}_{h} \cap \mathcal{Z}=\left\{\boldsymbol{v}_{h} \in \mathcal{N}_{h}: \operatorname{curl} \boldsymbol{v}_{h} \cdot \boldsymbol{n}=0 \text { on } \Gamma\right\} .
$$

The Galerkin approximation of Problem 3 reads as follows:

Problem 4. Find $\lambda_{h} \in \mathbb{C}$ and $\boldsymbol{u}_{h} \in \mathcal{Z}_{h}, \boldsymbol{u}_{h} \neq \mathbf{0}$, such that

$$
\int_{\Omega} \operatorname{curl} \boldsymbol{u}_{h} \cdot \operatorname{curl} \overline{\boldsymbol{v}}_{h}=\lambda_{h}^{2} \int_{\Omega} \boldsymbol{u}_{h} \cdot \overline{\boldsymbol{v}}_{h} \quad \forall \boldsymbol{v}_{h} \in \mathcal{Z}_{h} .
$$

Notice that Problem 4 leads to a well-posed generalized matrix eigenvalue problem, because the sesquilinear form on the right hand side is Hermitian positive definite. To solve this problem, it is necessary to impose somehow the constraint $\operatorname{curl} \boldsymbol{u}_{h} \cdot \boldsymbol{n}=0$ in the definition of $\mathcal{Z}_{h}$; we will address this point in Section 5 .

Consider the corresponding discrete solution operator:

$$
\begin{aligned}
T_{h}: \mathrm{L}^{2}(\Omega)^{3} & \longrightarrow \mathrm{L}^{2}(\Omega)^{3}, \\
\boldsymbol{f} & \longmapsto T_{h} \boldsymbol{f}:=\boldsymbol{w}_{h},
\end{aligned}
$$

with $\boldsymbol{w}_{h} \in \mathcal{Z}_{h}$ such that

$$
\int_{\Omega} \operatorname{curl} \boldsymbol{w}_{h} \cdot \operatorname{curl} \overline{\boldsymbol{v}}_{h}+\int_{\Omega} \boldsymbol{w}_{h} \cdot \overline{\boldsymbol{v}}_{h}=\int_{\Omega} \boldsymbol{f} \cdot \overline{\boldsymbol{v}}_{h} \quad \forall \boldsymbol{v}_{h} \in \mathcal{Z}_{h} .
$$

As a consequence of Lax Milgram lemma, $T_{h}$ is a well-defined bounded linear operator. Clearly $\lambda_{h}$ is an eigenvalue of Problem 4 if an only if $\frac{1}{1+\lambda_{h}^{2}} \in \sigma\left(T_{h}\right)$.

To prove convergence and error estimates for the proposed Galerkin scheme, we will use the results on spectral approximation for non-compact operators from $[12,13]$. With this aim, we consider the restrictions of the operators $T$ and $T_{h}$ to the respective invariant subspaces $\mathcal{Z}$ and $\mathcal{Z}_{h}$. To avoid overburdening the notation, from now on $T$ and $T_{h}$ will denote $\left.T\right|_{\mathcal{z}}$ and $\left.T_{h}\right|_{\mathcal{z}_{h}}$, respectively. Note that the spectral characterization of $T$ given in Theorem 3.9 remains the same without the need of any modification.

In order to use the theory from $[12,13]$ we need to prove the following properties:

P1: $\lim _{h \rightarrow 0}\left\|\left(T-T_{h}\right) \mid \mathcal{z}_{h}\right\|=0$,

P2: $\forall \boldsymbol{v} \in \mathcal{Z} \lim _{h \rightarrow 0} \inf _{\boldsymbol{v}_{h} \in \mathcal{Z}_{h}}\left\|\boldsymbol{v}-\boldsymbol{v}_{h}\right\|_{\mathbf{c u r l}, \Omega}=0$.

Property P2 follows immediately from Proposition 3.4 and standard interpolation error estimates for Nédélec finite elements. In order to prove property $\mathrm{P} 1$, we establish some preliminary results.

Let us define

$$
r_{k}:=\min \{s, k\},
$$

where $k \geq 1$ is the degree of the Nédélec finite elements and $s>1 / 2$ is a Sobolev exponent such that $(2.1)$ holds true.

Let us recall that $\mathcal{K} \subset \mathcal{Z}$ is the eigenspace of $T$ associated with the eigenvalue $\mu=1$ and let $\mathcal{V}:=\mathcal{K}^{\perp \mathcal{z}}$. It is immediate to show from (3.4) that $\mathcal{V}=\mathrm{H}_{0}\left(\operatorname{div}^{0} ; \Omega\right) \cap$ $\mathcal{Z}$. Operator $T$ restricted to $\mathcal{K}$ is the identity; instead, restricted to its orthogonal complement is a regularizing operator, as shown in the following lemma.

Lemma 4.1. Let $\boldsymbol{f} \in \mathcal{V}$ and $\boldsymbol{w}:=T \boldsymbol{f}$. Then, $\boldsymbol{w} \in \mathrm{H}^{s}(\operatorname{curl} ; \Omega)$ and

$$
\|\boldsymbol{w}\|_{s, \Omega}+\|\operatorname{curl} \boldsymbol{w}\|_{s, \Omega} \leq C\|\boldsymbol{f}\|_{0, \Omega} .
$$


Proof. From the definition of $T, \boldsymbol{w}$ and $\boldsymbol{f}$ are related by (3.3). Taking $\boldsymbol{v}=\boldsymbol{w}$ in this equation, we have

$$
\|\boldsymbol{w}\|_{\operatorname{curl}, \Omega} \leq\|\boldsymbol{f}\|_{0, \Omega} .
$$

Moreover, since $\nabla\left(\mathrm{H}^{1}(\Omega)\right) \subset \mathcal{Z}$, taking in $(3.3) \boldsymbol{v}=\nabla \psi, \psi \in \mathrm{H}^{1}(\Omega)$, it follows that

$$
\int_{\Omega} \boldsymbol{w} \cdot \nabla \bar{\psi}=\int_{\Omega} \boldsymbol{f} \cdot \nabla \bar{\psi}=0 \quad \forall \psi \in \mathrm{H}^{1}(\Omega) .
$$

Hence, for $\boldsymbol{f} \in \mathcal{V} \subset \mathrm{H}_{0}\left(\operatorname{div}^{0} ; \Omega\right)$, we have that $\boldsymbol{w} \in \mathrm{H}_{0}\left(\operatorname{div}^{0} ; \Omega\right)$, too. Consequently, $\boldsymbol{w} \in \mathrm{H}(\operatorname{curl} ; \Omega) \cap \mathrm{H}_{0}\left(\operatorname{div}^{0} ; \Omega\right) \hookrightarrow \mathrm{H}^{s}(\Omega)^{3}$ (cf. (2.1)) and, using (4.2), it follows that

$$
\|\boldsymbol{w}\|_{s, \Omega} \leq C\|\boldsymbol{w}\|_{\operatorname{curl}, \Omega} \leq C\|\boldsymbol{f}\|_{0, \Omega} .
$$

On the other hand, taking $\boldsymbol{v} \in \mathcal{D}(\Omega)^{3} \subset \mathcal{Z}$ in (3.3), we obtain

$$
\operatorname{curl}(\operatorname{curl} \boldsymbol{w})+\boldsymbol{w}=\boldsymbol{f} \text { in } \Omega \text {. }
$$

Hence, $\operatorname{curl} \boldsymbol{w} \in \mathrm{H}(\operatorname{curl} ; \Omega)$ and, since $\boldsymbol{w} \in \mathcal{Z}, \operatorname{curl} \boldsymbol{w} \in \mathrm{H}_{0}\left(\operatorname{div}^{0} ; \Omega\right)$, too. Then, the same arguments as above allow us to conclude that $\operatorname{curl} \boldsymbol{w} \in \mathrm{H}^{s}(\Omega)^{3}$ and

$$
\|\operatorname{curl} \boldsymbol{w}\|_{s, \Omega} \leq C\|\operatorname{curl} \boldsymbol{w}\|_{\operatorname{curl}, \Omega} \leq C\|\boldsymbol{f}\|_{0, \Omega},
$$

the last inequality because of (4.3) and (4.2). Thus, we conclude the proof.

Clearly $\mu_{h}=1$ is an eigenvalue of $T_{h}$ with associated eigenspace

$$
\mathcal{K}_{h}:=\left\{\boldsymbol{v}_{h} \in \mathcal{Z}_{h}: \operatorname{curl} \boldsymbol{v}_{h}=\mathbf{0}\right\} \subset \mathcal{K},
$$

so that $T_{h}$ restricted to $\mathcal{K}_{h}$ is the identity, too. Let $\mathcal{V}_{h}:=\mathcal{K}_{h}^{\perp \boldsymbol{z}_{h}}$. Notice that $\mathcal{V}_{h} \not \subset \mathcal{V}$. However, the following lemma shows that the curl-free terms in the Helmholtz decomposition of $\mathcal{V}_{h}$ are asymptotically negligible.

Lemma 4.2. For $\boldsymbol{f}_{h} \in \mathcal{V}_{h}$, there exist $\chi \in \mathcal{V}$ and $\boldsymbol{\eta} \in \mathcal{K}$ such that $\boldsymbol{f}_{h}=\boldsymbol{\chi}+\boldsymbol{\eta}$ and there hold:

a) $\boldsymbol{\chi} \in \mathrm{H}^{s}(\Omega)^{3}$ with $\|\boldsymbol{\chi}\|_{s, \Omega} \leq C\left\|\operatorname{curl} \boldsymbol{f}_{h}\right\|_{0, \Omega}$,

b) $\|\boldsymbol{\eta}\|_{0, \Omega} \leq C h^{r_{1}}\left\|\operatorname{curl} \boldsymbol{f}_{h}\right\|_{0, \Omega}$, with $r_{1}$ as defined in (4.1).

Proof. Since $\boldsymbol{f}_{h} \in \mathcal{V}_{h} \subset \mathcal{Z}$, the decomposition $\boldsymbol{f}_{h}=\boldsymbol{\chi}+\boldsymbol{\eta}$ follows from the fact that $\mathcal{V}=\mathcal{K}^{\perp \mathcal{z}}$. Now, since $\mathcal{V} \subset \mathrm{H}(\operatorname{curl} ; \Omega) \cap \mathrm{H}_{0}\left(\operatorname{div}^{0} ; \Omega\right)$, we have that $\boldsymbol{\chi} \in \mathrm{H}^{s}(\Omega)^{3}$ (cf. (2.1), again). Moreover, because of the definition of $\mathcal{K}, \operatorname{curl} \chi=\operatorname{curl} \boldsymbol{f}_{h}$. Hence,

$$
\|\chi\|_{s, \Omega} \leq C\|\chi\|_{\operatorname{curl}, \Omega} \leq C\|\operatorname{curl} \chi\|_{0, \Omega}=C\left\|\operatorname{curl} \boldsymbol{f}_{h}\right\|_{0, \Omega},
$$

the last inequality because, for $\Omega$ simply-connected, $\|\chi\|_{0, \Omega} \leq C\|\operatorname{curl} \chi\|_{0, \Omega}$ for all $\chi \in \mathrm{H}(\operatorname{curl} ; \Omega) \cap \mathrm{H}_{0}\left(\operatorname{div}^{0} ; \Omega\right)$ (cf. [1, Corollary 3.16]). Thus we conclude (a).

To prove (b), we will use the Nédélec interpolant $I_{h}^{N}$. According to $[18$, Theorem 5.41(2)], since $\operatorname{curl} \boldsymbol{\chi}=\operatorname{curl} \boldsymbol{f}_{h}$, we have that

$$
\left\|\chi-I_{h}^{N} \chi\right\|_{0, \Omega} \leq C\left(h^{r_{1}}\|\chi\|_{s, \Omega}+h\left\|\operatorname{curl} \boldsymbol{f}_{h}\right\|_{0, \Omega}\right) \leq C h^{r_{1}}\left\|\operatorname{curl} \boldsymbol{f}_{h}\right\|_{0, \Omega} .
$$

On the other hand, let $I_{h}^{R}$ be the divergence-conforming Raviart-Thomas interpolant (see [18, Section 5.4]). Since $\operatorname{curl} \boldsymbol{\chi}=\operatorname{curl} \boldsymbol{f}_{h} \in \mathrm{H}^{\varepsilon}(\Omega)^{3}$ for all $\varepsilon \in\left(0, \frac{1}{2}\right)$, according to Remark 5.16 and Lemma 5.40 from [18], it follows that

$$
\operatorname{curl}\left(I_{h}^{N} \chi\right)=I_{h}^{R}(\operatorname{curl} \chi)=I_{h}^{R}\left(\operatorname{curl} \boldsymbol{f}_{h}\right)=\operatorname{curl} \boldsymbol{f}_{h} .
$$


Now, we write

$$
\|\boldsymbol{\eta}\|_{0, \Omega}^{2}=\int_{\Omega} \boldsymbol{\eta} \cdot\left(\boldsymbol{f}_{h}-\boldsymbol{\chi}\right)=\int_{\Omega} \boldsymbol{\eta} \cdot\left(\boldsymbol{f}_{h}-I_{h}^{N} \boldsymbol{\chi}\right)+\int_{\Omega} \boldsymbol{\eta} \cdot\left(I_{h}^{N} \boldsymbol{\chi}-\boldsymbol{\chi}\right) .
$$

By virtue of (4.5) we have that $\boldsymbol{f}_{h}-I_{h}^{N} \boldsymbol{\chi} \in \mathcal{K}_{h} \subset \mathcal{K}$, so that $\chi \perp\left(\boldsymbol{f}_{h}-I_{h}^{N} \boldsymbol{\chi}\right)$ in $\mathrm{L}^{2}(\Omega)^{3}$ and, since $\boldsymbol{f}_{h} \in \mathcal{V}_{h}=\mathcal{K}_{h}^{\perp_{z_{h}}}$, it follows that

$$
\int_{\Omega} \boldsymbol{\eta} \cdot\left(\boldsymbol{f}_{h}-I_{h}^{N} \boldsymbol{\chi}\right)=\int_{\Omega} \boldsymbol{f}_{h} \cdot\left(\boldsymbol{f}_{h}-I_{h}^{N} \boldsymbol{\chi}\right)-\int_{\Omega} \boldsymbol{\chi} \cdot\left(\boldsymbol{f}_{h}-I_{h}^{N} \boldsymbol{\chi}\right)=0 .
$$

Hence, from (4.6) and (4.4), we conclude that

$$
\|\boldsymbol{\eta}\|_{0, \Omega} \leq\left\|I_{h}^{N} \chi-\chi\right\|_{0, \Omega} \leq C h^{r_{1}}\left\|\operatorname{curl} \boldsymbol{f}_{h}\right\|_{0, \Omega} .
$$

Thus, we end the proof.

Now we are ready to prove the following result, from which we will derive property P1.

Lemma 4.3. There exists $C>0$ such that, for all $\boldsymbol{f}_{h} \in \mathcal{V}_{h}$,

$$
\left\|\left(T-T_{h}\right) \boldsymbol{f}_{h}\right\|_{\mathbf{c u r l}, \Omega} \leq C h^{r_{1}}\left\|\boldsymbol{f}_{h}\right\|_{\mathbf{c u r l}, \Omega},
$$

with $r_{1}$ as defined in (4.1).

Proof. Given $\boldsymbol{f}_{h} \in \mathcal{V}_{h}$, let $\boldsymbol{\chi} \in \mathcal{V}$ and $\boldsymbol{\eta} \in \mathcal{K}$ be as in Lemma 4.2. Let $\boldsymbol{z}:=T \boldsymbol{\chi}$ and $z_{h}:=T_{h} \boldsymbol{\chi}$. The following Cea estimate follows immediately from the definitions of $T$ and $T_{h}$ :

$$
\left\|\boldsymbol{z}-\boldsymbol{z}_{h}\right\|_{\mathbf{c u r l}, \Omega} \leq C \inf _{\boldsymbol{v}_{h} \in \mathcal{Z}_{h}}\left\|\boldsymbol{z}-\boldsymbol{v}_{h}\right\|_{\mathbf{c u r l}, \Omega}
$$

Then, using the Nédélec interpolant and standard error estimates (cf. [18, Theorem 5.41(1)]), it follows that

$$
\left\|\boldsymbol{z}-\boldsymbol{z}_{h}\right\|_{\operatorname{curl}, \Omega} \leq C\left\|\boldsymbol{z}-I_{h}^{N} \boldsymbol{z}\right\|_{\operatorname{curl}, \Omega} \leq C h^{r_{k}}\left(\|\boldsymbol{z}\|_{s, \Omega}+\|\operatorname{curl} \boldsymbol{z}\|_{s, \Omega}\right) .
$$

Thus, from Lemma 4.1, and the fact that $\mathcal{K} \perp \mathcal{V}$ in $\mathrm{L}^{2}(\Omega)$, we have

$$
\left\|\left(T-T_{h}\right) \chi\right\|_{\operatorname{curl}, \Omega}=\left\|\boldsymbol{z}-\boldsymbol{z}_{h}\right\|_{\operatorname{curl}, \Omega} \leq C h^{r_{k}}\|\chi\|_{0, \Omega} \leq C h^{r_{k}}\left\|\boldsymbol{f}_{h}\right\|_{0, \Omega} .
$$

On the other hand, for $\eta \in \mathcal{K}$, since $T \boldsymbol{\eta}=\boldsymbol{\eta}$ and $T_{h} \boldsymbol{\eta}$ is the Galerkin projection of $\boldsymbol{\eta}$ onto $\mathcal{Z}_{h}$, using Lemma 4.2(b) we can write

$$
\left\|\left(T-T_{h}\right) \boldsymbol{\eta}\right\|_{\mathbf{c u r l}, \Omega} \leq\|\boldsymbol{\eta}\|_{\operatorname{curl}, \Omega}=\|\boldsymbol{\eta}\|_{0, \Omega} \leq C h^{r_{1}}\left\|\boldsymbol{f}_{h}\right\|_{\mathbf{c u r l}, \Omega} .
$$

Therefore,

$$
\left\|\left(T-T_{h}\right) \boldsymbol{f}_{h}\right\|_{\mathbf{c u r l}, \Omega} \leq\left\|\left(T-T_{h}\right) \boldsymbol{\chi}\right\|_{\mathbf{c u r l}, \Omega}+\left\|\left(T-T_{h}\right) \boldsymbol{\eta}\right\|_{\mathbf{c u r l}, \Omega} \leq C h^{r_{1}}\left\|\boldsymbol{f}_{h}\right\|_{\mathbf{c u r l}, \Omega}
$$

and we conclude the proof.

Property P1 clearly follows from the above lemma and the fact that $T$ and $T_{h}$ coincide on $\mathcal{K}_{h}$. As a first consequence, we have the next result, which was proved to follow from property P1 in [12, Theorem 1].

Theorem 4.4. Let $J \subset \mathbb{R}$ be an open set containing $\sigma(T)$. Then, there exists $h_{0}>0$ such that $\sigma\left(T_{h}\right) \subset J \forall h<h_{0}$. 
As a consequence of the above theorem, we know that the proposed numerical method does not introduce spurious modes (which would be the case, for instance, if Lagrangian finite elements were used; see [4]).

Now, we are in a position to write the main result of this paper related to the convergence of the proposed scheme.

Theorem 4.5. Let $\mu \in \sigma(T)$ be an eigenvalue of finite-multiplicity $m$. Let $\mathcal{E}$ be the corresponding eigenspace. There exists $h_{0}>0$ such that, for all $h<h_{0}$, $\sigma\left(T_{h}\right)$ contains $m$ eigenvalues $\mu_{h}^{(1)}, \ldots, \mu_{h}^{(m)}$ (repeated accordingly to their respective multiplicities) such that

$$
\mu_{h}^{(i)} \underset{h \rightarrow 0}{\longrightarrow} \mu, \quad i=1, \ldots, m .
$$

Let $\mathcal{E}_{h}$ be the direct sum of the corresponding eigenspaces. Then,

$$
\widehat{\delta}\left(\mathcal{E}, \mathcal{E}_{h}\right) \leq C \gamma_{h},
$$

and

$$
\max _{1 \leq i \leq m}\left|\mu-\mu_{h}^{(i)}\right| \leq C \gamma_{h}^{2}
$$

where

$$
\gamma_{h}:=\delta\left(\mathcal{E}, \mathcal{Z}_{h}\right):=\sup _{\substack{\boldsymbol{v} \in \mathcal{E} \\\|\boldsymbol{v}\|_{\mathbf{c u r l}, \Omega}=1}} \inf _{\boldsymbol{v}_{h} \in \mathcal{Z}_{h}}\left\|\boldsymbol{v}-\boldsymbol{v}_{h}\right\|_{\mathbf{c u r l}, \Omega}
$$

and

$$
\widehat{\delta}\left(\mathcal{E}, \mathcal{E}_{h}\right):=\max \left\{\delta\left(\mathcal{E}, \mathcal{E}_{h}\right), \delta\left(\mathcal{E}_{h}, \mathcal{E}\right)\right\} .
$$

Proof. Since we have already proved that properties P1 and P2 hold true, the results are direct consequences of [12, Section 2] and Theorems 1 and 3 from [13].

To conclude spectral convergence with an optimal order of approximation from the previous theorem, we only need an appropriate estimate for the term $\gamma_{h}$.

Theorem 4.6. Let $\gamma_{h}$ be as in Theorem 4.5. Then, there exists $C>0$ such that

$$
\gamma_{h} \leq C h^{r_{k}},
$$

with $r_{k}$ as defined in (4.1).

Proof. Let $\boldsymbol{v} \in \mathcal{E}$ be such that $\|\boldsymbol{v}\|_{\mathbf{c u r l}, \Omega}=1$. Since $T \boldsymbol{v}=\mu \boldsymbol{v}$, from Lemma 4.1 it follows that $\boldsymbol{v} \in \mathrm{H}^{s}(\operatorname{curl} ; \Omega)$ and

$$
\|\boldsymbol{v}\|_{s, \Omega}+\|\operatorname{curl} \boldsymbol{v}\|_{s, \Omega} \leq \frac{C}{\mu}\|\boldsymbol{v}\|_{0, \Omega} \leq \frac{C}{\mu} .
$$

Let $I_{h}^{N} \boldsymbol{v} \in \mathcal{N}_{h}$ be the Nédélec interpolant of $\boldsymbol{v}$; in what follows, we show that $I_{h}^{N} \boldsymbol{v} \in \mathcal{Z}_{h}$ (this has been proved in [16, Proposition 4.3] and [3, Lemma 2.2], but only for lowest-order Nédélec elements and under different topological assumptions). Let $I_{h}^{R}$ be the divergence-conforming Raviart-Thomas interpolant. Since $\boldsymbol{v} \in \mathcal{E} \subset$ $\mathcal{Z}$, we have that $\operatorname{curl} \boldsymbol{v} \cdot \boldsymbol{n}=0$ on $\Gamma$. Hence, $\operatorname{curl}\left(I_{h}^{N} \boldsymbol{v}\right) \cdot \boldsymbol{n}=\left(I_{h}^{R} \operatorname{curl} \boldsymbol{v}\right) \cdot \boldsymbol{n}=0$ on $\Gamma$, too, the first equality because of [18, Lemma 5.40] and the second one because of the well known property that the Raviart-Thomas interpolant preserves vanishing normal components on the boundary. Thus, $I_{h}^{N} \boldsymbol{v} \in \mathcal{Z} \cap \mathcal{N}_{h}=\mathcal{Z}_{h}$. 
Therefore, using again the standard error estimate for the Nédélec interpolant (cf. [18, Theorem 5.41(1)]), we obtain

$$
\delta\left(\mathcal{E}, \mathcal{Z}_{h}\right) \leq \sup _{\substack{\boldsymbol{v} \in \mathcal{E} \\\|\boldsymbol{v}\|_{\text {curl }, \Omega}=1}}\left\|\boldsymbol{v}-I_{h}^{N} \boldsymbol{v}\right\|_{\operatorname{curl}, \Omega} \leq C h^{r_{k}}\left(\|\boldsymbol{v}\|_{s, \Omega}+\|\operatorname{curl} \boldsymbol{v}\|_{s, \Omega}\right) \leq \frac{C}{\mu} h^{r_{k}} .
$$

Thus, we end the proof.

As a consequence of the two previous theorems we conclude that the eigenvalues and eigenfunctions of Problem 4 converge with optimal order to those of Problem 3.

\section{IMPLEMENTATION ISSUES}

For the implementation of Problem 4, it is necessary to impose the condition $\operatorname{curl} \boldsymbol{u}_{h} \cdot \boldsymbol{n}=0$ on $\Gamma$. To do this, we follow a similar approach to that used in [16] and [3] for lowest-order Nédélec elements.

Since we have assumed that the domain $\Omega$ is simply-connected, each connected component of its boundary is simply-connected, too. In such a case, $\operatorname{curl} \boldsymbol{u}_{h} \cdot \boldsymbol{n}=0$ on $\Gamma$ if and only if the tangential component of $\boldsymbol{u}_{h}$ satisfies

$$
\boldsymbol{n} \times \boldsymbol{u}_{h} \times \boldsymbol{n}=\nabla_{\Gamma} \varphi_{h} \quad \text { on } \Gamma,
$$

where $\varphi_{h} \in \mathcal{L}_{h}^{\Gamma}:=\left\{\psi_{h} \in \mathcal{C}(\Gamma):\left.\psi_{h}\right|_{F} \in \mathbb{P}_{k}(F) \forall F \in \mathcal{T}_{h}^{\Gamma}\right\}$ and $\nabla_{\Gamma}$ denotes the surface gradient (i.e., the two-dimensional gradient on each plane face of $\Gamma$; see [8] for its proper definition). In fact, it is shown in [8, Section 4] that $\operatorname{curl} \boldsymbol{u}_{h} \cdot \boldsymbol{n}=$ $\operatorname{curl}_{\Gamma}\left(\boldsymbol{n} \times \boldsymbol{u}_{h} \times \boldsymbol{n}\right)$ on $\Gamma$, where $\operatorname{curl}_{\Gamma}$ denotes the scalar surface curl. Hence, from [8, Theorem 5.1], we know that there exists $\varphi_{h} \in \mathrm{H}^{1}(\Gamma)$ such that $\boldsymbol{n} \times \boldsymbol{u}_{h} \times \boldsymbol{n}=\nabla_{\Gamma} \varphi_{h}$ on $\Gamma$. Moreover, by using [18, Remark 5.29], it is easy to show that $\varphi_{h} \in \mathcal{L}_{h}^{\Gamma}$.

Let

$$
\mathcal{L}_{h}:=\left\{\psi_{h} \in \mathcal{C}(\Omega):\left.\psi_{h}\right|_{T} \in \mathbb{P}_{k}(T) \quad \forall T \in \mathcal{T}_{h}\right\}
$$

Let $\left\{\varphi_{j}\right\}_{j=1}^{K}$ be the nodal basis of $\mathcal{L}_{h}$. Without loss of generality we order these basis functions so that the first $J$ of them correspond to all the nodal values on the boundary $\Gamma$. Therefore $\left\{\left.\varphi_{j}\right|_{\Gamma}\right\}_{j=1}^{J}$ is a basis of $\mathcal{L}_{h}^{\Gamma}$. Moreover, $\left\langle\left\{\nabla_{\Gamma} \varphi_{j}\right\}_{j=1}^{J}\right\rangle=$ $\nabla_{\Gamma}\left(\mathcal{L}_{h}^{\Gamma}\right)$. However, these functions are not linearly independent. To obtain a basis of $\nabla_{\Gamma}\left(\mathcal{L}_{h}^{\Gamma}\right)$, we must choose one vertex on each connected component $\Gamma_{0}, \ldots, \Gamma_{I}$ of $\Gamma$ and drop out the basis function corresponding to these vertices. Let us assume for simplicity that these basis function are the last ones. Then, it is straightforward to show that $\left\{\nabla_{\Gamma} \varphi_{j}\right\}_{j=1}^{L}(L:=J-I-1)$ is a basis of $\nabla_{\Gamma}\left(\mathcal{L}_{h}^{\Gamma}\right)$.

Let $\left\{\phi_{m}\right\}_{m=1}^{M}$ be the nodal basis of $\mathcal{N}_{h}$; without loss of generality we also assume that the last ones, $\left\{\boldsymbol{\phi}_{m}\right\}_{m=N+1}^{M}$, are those corresponding to the degrees of freedom related to the faces or edges on $\Gamma$. Notice that all the other basis functions lie in $\mathcal{Z}_{h}$. Thus, we have the following proposition that characterizes a basis of this space.

Proposition 5.1. The set $\left\{\boldsymbol{\phi}_{m}\right\}_{m=1}^{N} \cup\left\{\nabla \varphi_{j}\right\}_{j=1}^{L}$ is a basis of $\mathcal{Z}_{h}$.

Proof. It is essentially identical to that of Proposition 4.2 from [16], where a similar result is proved in the case that $\Gamma$ is connected for lowest-order Nédélec elements. We include it for completeness. 
First we prove that $\left\{\phi_{m}\right\}_{m=1}^{N} \cup\left\{\nabla \varphi_{j}\right\}_{j=1}^{L}$, which is clearly a subset of $\mathcal{Z}_{h}$, spans this space. Let $\phi_{h} \in \mathcal{Z}_{h}$. Because of (5.1), $\boldsymbol{n} \times\left.\boldsymbol{\phi}_{h}\right|_{\Gamma} \times \boldsymbol{n} \in \nabla_{\Gamma}\left(\mathcal{L}_{h}^{\Gamma}\right)$ and, hence, there exist $\beta_{j}, j=1, \ldots, L$, such that

$$
\boldsymbol{n} \times\left.\boldsymbol{\phi}_{h}\right|_{\Gamma} \times \boldsymbol{n}=\sum_{j=1}^{L} \beta_{j} \nabla_{\Gamma} \varphi_{j} .
$$

Then, the degrees of freedom of $\phi_{h}-\sum_{j=1}^{L} \beta_{j} \nabla \varphi_{j} \in \mathcal{N}_{h}$ corresponding to edges or faces lying on the boundary vanish. Therefore,

$$
\phi_{h}-\sum_{j=1}^{L} \beta_{j} \nabla \varphi_{j} \in\left\langle\phi_{1}, \ldots, \phi_{N}\right\rangle .
$$

It only remains to prove that $\left\{\boldsymbol{\phi}_{m}\right\}_{m=1}^{N} \cup\left\{\nabla \varphi_{j}\right\}_{j=1}^{L}$ is a linearly independent set. Let us assume that

$$
\sum_{m=1}^{N} \alpha_{m} \phi_{m}+\sum_{j=1}^{L} \beta_{j} \nabla \varphi_{j}=0 .
$$

Since $\boldsymbol{n} \times\left.\boldsymbol{\phi}_{m}\right|_{\Gamma} \times \boldsymbol{n}$ vanish for all $m=1, \ldots, N$ and $\boldsymbol{n} \times\left.\nabla \varphi_{j}\right|_{\Gamma} \times \boldsymbol{n}=\nabla_{\Gamma} \varphi_{j}$ is a basis of $\nabla_{\Gamma}\left(\mathcal{L}_{h}^{\Gamma}\right)$, we have that $\beta_{1}=\cdots=\beta_{L}=0$. Thus the result follows from the linear independence of $\left\{\phi_{m}\right\}_{m=1}^{N}$.

Actually, the constraint $\operatorname{curl} \boldsymbol{u}_{h} \cdot \boldsymbol{n}=0$ on $\Gamma$ in the definition of $\mathcal{Z}_{h}$ can be imposed without the need of using the basis functions $\left\{\nabla \varphi_{j}\right\}_{j=1}^{L}$. We illustrate this in the case of lowest-order Nédélec elements, which are the ones that we have implemented in the code used for the numerical tests reported in the next section.

Let $\left\{e_{1}, \ldots, e_{M}\right\}$ be the set of all edges in $\mathcal{T}_{h}$ and $\left\{\boldsymbol{\phi}_{m}\right\}_{m=1}^{M}$ be the associated nodal basis of $\mathcal{N}_{h}$. Then, for any $\boldsymbol{u}_{h} \in \mathcal{N}_{h}$,

$$
\boldsymbol{u}_{h}=\sum_{m=1}^{M} \alpha_{m} \boldsymbol{\phi}_{m} \text {. }
$$

where $\alpha_{m}:=\int_{e_{m}} \boldsymbol{u}_{h} \cdot \boldsymbol{t}_{m}$, with $\boldsymbol{t}_{m}$ a unit tangent to $e_{m}, m=1, \ldots, M$. We assume as above that the edges lying on $\Gamma$ are the last ones: $e_{N+1}, \ldots, e_{M}$.

Let $\left\{P_{j}\right\}_{j=1}^{J}$ be the set of vertices of $\mathcal{T}_{h}$ lying on $\Gamma$, where the last $I+1$ have been chosen one on each connected component of $\Gamma$, also as above. Let $\left\{\varphi_{j}\right\}_{j=1}^{J}$ be the corresponding nodal basis of $\mathcal{L}_{h}^{\Gamma}$. In such a case, according to the Proposition 5.1, for $\boldsymbol{u}_{h} \in \mathcal{Z}_{h}$, there exist complex numbers $\alpha_{1}^{\prime}, \ldots, \alpha_{N}^{\prime}$ and $\beta_{1}, \ldots, \beta_{J}$ such that

$$
\boldsymbol{u}_{h}=\sum_{m=1}^{N} \alpha_{m}^{\prime} \boldsymbol{\phi}_{m}+\sum_{j=1}^{J} \beta_{j} \nabla \varphi_{j},
$$

where $\beta_{L+1}=\cdots=\beta_{J}=0$. Then, from the definition of $\alpha_{m}$ and the above relation, we obtain:

$$
\alpha_{m}= \begin{cases}\alpha_{m}^{\prime}, & \text { if } e_{m} \cap \Gamma=\emptyset, \\ \alpha_{m}^{\prime} \pm \beta_{j}, & \text { if } e_{m} \cap \Gamma=\left\{P_{j}\right\} \\ \alpha_{m}^{\prime} \pm\left(\beta_{j}-\beta_{k}\right), & \text { if } e_{m} \cap \Gamma=\left\{P_{j}, P_{k}\right\} \quad\left(e_{m} \not \subset \Gamma\right), \\ \pm\left(\beta_{j}-\beta_{k}\right), & \text { if } e_{m} \subset \Gamma, \text { with end points } P_{j}, P_{k},\end{cases}
$$


the signs above depend on the chosen orientation of the tangent vector $\boldsymbol{t}_{m}$.

These relations allow us to define a matrix $\boldsymbol{C} \in \mathbb{R}^{M \times(N+L)}$ such that $\boldsymbol{\alpha}=\boldsymbol{C} \widehat{\boldsymbol{\alpha}}$, where $\boldsymbol{\alpha}:=\left(\alpha_{1}, \ldots, \alpha_{M}\right)^{\mathrm{t}}$ and $\widehat{\boldsymbol{\alpha}}:=\left(\alpha_{1}^{\prime}, \ldots, \alpha_{N}^{\prime}, \beta_{1}, \ldots, \beta_{L}\right)^{\mathrm{t}}$. Notice that most of the entries of this matrix vanish and the others are \pm 1 .

Let $\boldsymbol{A}:=\left(A_{i j}\right)$ and $\boldsymbol{B}:=\left(B_{i j}\right)$ be the $M \times M$ matrices defined by

$$
A_{i j}:=\int_{\Omega} \operatorname{curl} \phi_{j} \cdot \operatorname{curl} \bar{\phi}_{i} \quad \text { and } \quad B_{i j}:=\int_{\Omega} \phi_{j} \cdot \bar{\phi}_{i}, \quad i, j=1, \ldots, M .
$$

Then, using the basis of $\mathcal{Z}_{h}$ from Proposition 5.1, the matrix form of Problem 4 reads as follows:

$$
\widehat{\boldsymbol{A}} \widehat{\boldsymbol{\alpha}}=\lambda_{h}^{2} \widehat{\boldsymbol{B}} \widehat{\boldsymbol{\alpha}},
$$

with Hermitian matrices $\widehat{\boldsymbol{A}}:=\boldsymbol{C}^{\mathrm{t}} \boldsymbol{B} \boldsymbol{C}$ and $\widehat{\boldsymbol{B}}:=\boldsymbol{C}^{\mathrm{t}} \boldsymbol{A} \boldsymbol{C}$, which are positive semidefinite and positive definite, respectively. Thus, this is a well-posed generalized matrix eigenvalue problem.

\section{NumERICAL EXPERIMENTS}

We have developed a MATLAB code based on lowest-order Nédélec elements $(k=1)$ to solve Problem 4 . We report in this section some numerical experiments which confirm the theoretical results proved in the previous sections.

6.1. Validation. As a first numerical test, we have solved a particular problem with a known analytical solution, which allowed us to validate the computer code and to check the performance and convergence properties of the scheme. When the domain $\Omega$ is the unit sphere, the least positive eigenvalue is the smallest positive solution of the equation $\lambda=\tan \lambda$, namely, $\lambda=4.493409 \ldots$ Moreover, $\lambda$ is an eigenvalue of multiplicity three (for further details see $[9$, Theorem A]).

Because of the symmetry of the domain, it is easy to check that $(\lambda, \boldsymbol{u}(\boldsymbol{x}))$ is a solution of Problem 1 if and only if $(-\lambda, \boldsymbol{u}(-\boldsymbol{x}))$ is a solution, too. Therefore, $\lambda^{2}$ is an eigenvalue of Problem 3 with multiplicity six. Whence, by virtue of Theorem 4.5, we know that, for $h$ small enough, there exist six eigenvalues $\lambda_{h, 1}^{2}, \ldots, \lambda_{h, 6}^{2}$ of Problem 4 (repeated accordingly to their respective multiplicities) such that

$$
\lambda_{h, i}^{2} \underset{h \rightarrow 0}{\longrightarrow} \lambda^{2}, \quad i=1, \ldots, 6 .
$$

The code has been used on several meshes $\mathcal{T}_{h}$ with different levels of refinement; we identify each mesh by its respective number of tetrahedra $N_{h}$. We have compared the average $\widehat{\lambda}_{h}:=\left(\lambda_{h, 1}+\ldots+\lambda_{h, 6}\right) / 6$ with the analytical eigenvalue $\lambda$. Table 1 shows the obtained results. The table also includes an estimate of the order of convergence, the so-called experimental rate of convergence:

$$
\operatorname{erc}:=-3 \frac{\log \left(\left|\lambda-\widehat{\lambda}_{h}\right| /\left|\lambda-\widehat{\lambda}_{h^{\prime}}\right|\right)}{\log \left(N_{h} / N_{h^{\prime}}\right)} .
$$

Since the domain is smooth, the theoretical order of convergence for the eigenvalues is in this case $\mathcal{O}\left(h^{2 r_{1}}\right)$, with $r_{1}:=\min \{s, 1\}=1$. It can be seen from Table 1 that the obtained results show an estimated order of convergence close to the theoretical one. Figure 1 shows a log-log plot of the errors versus the number of tetrahedra $N_{h}$. The slope of the line shows a clear quadratic dependence on the mesh-size. 
TABLE 1. Unit sphere. Computed and exact eigenvalues, errors, and experimental rates of convergence.

\begin{tabular}{c|ccc|c}
$N_{h}$ & $\widehat{\lambda}_{h}$ & $\lambda$ & $\left|\lambda-\widehat{\lambda}_{h}\right|$ & erc \\
\hline 53506 & 4.495885 & 4.493409 & 0.002475 & - \\
91286 & 4.495117 & 4.493409 & 0.001708 & 2.08 \\
157765 & 4.494620 & 4.493409 & 0.001210 & 1.89 \\
259404 & 4.494283 & 4.493409 & 0.000874 & 1.96
\end{tabular}

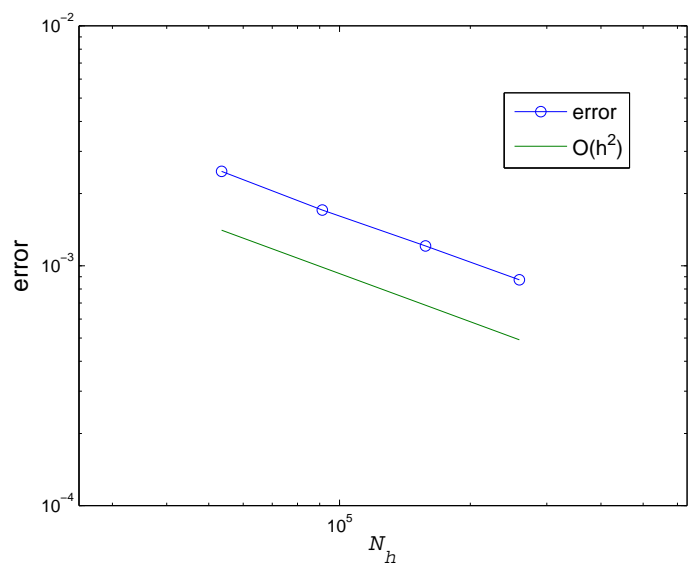

FIGURE 1. Unit sphere. Error $\left|\lambda-\widehat{\lambda}_{h}\right|$ versus number of tetrahedra $N_{h}$ (log-log scale).

According to the theoretical results, the invariant subspace spanned by the six eigenfunctions of Problem 4 corresponding to $\lambda_{h, 1}, \ldots, \lambda_{h, 6}$ yields an approximation of the eigenspace of $\lambda^{2}$ in Problem 3. However, the latter is the direct sum of two three-dimensional eigenspaces of Problem 1, those corresponding to $\lambda$ and $-\lambda$. Therefore, the eigenfunctions of Problem 4 are not in general eigenfunctions of Problem 1 , but linear combination of eigenfunctions corresponding to both eigenvalues, $\lambda$ and $-\lambda$. Because of this, the present method does not necessarily furnish approximations of the eigenfunctions. To compute the eigenfunctions we have solved numerically Problem 2. With this aim, we have used the finite element discretization given in Problem 6. More details about this numerical method, including the corresponding convergence analysis, are reported in the appendix.

Figure 2 shows the vector field and some integral curves for one of the eigenfunctions. This minimum-eigenvalue field is the well-known spheromak introduced in [11] (see also [10, 23, 24]) and reported in [9].

We have also applied our method to a problem in which the boundary of the domain is not connected: a spherical shell $\left\{\boldsymbol{x} \in \mathbb{R}^{3}: a \leq|\boldsymbol{x}| \leq b\right\}$. In this case, two basis functions of $\mathcal{L}_{h}^{\Gamma}$ has to be eliminated for the implementation, each of them corresponding to a vertex on each connected component.

We have compared the obtained results with the analytical ones reported in [9] for the spherical shell $\left\{\boldsymbol{x} \in \mathbb{R}^{3}: 0.540183 \leq|\boldsymbol{x}| \leq 1.05\right\}$. Table 2 shows results 


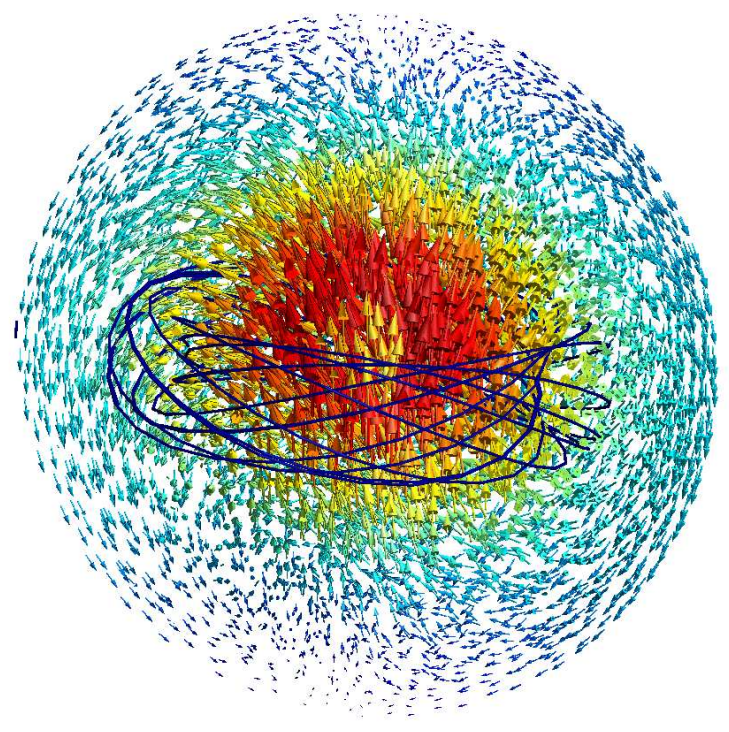

FiguRE 2. The spheromak.

similar to those reported in Table 1 for the eigenvalue of least absolute value of this problem. Once more, the estimated rates of convergence are close to $\mathcal{O}\left(h^{2}\right)$.

TABle 2. Spherical shell. Computed and exact eigenvalues, errors, and experimental rates of convergence.

\begin{tabular}{c|ccc|c}
$N_{h}$ & $\widehat{\lambda}_{h}$ & $\lambda$ & $\left|\lambda-\widehat{\lambda}_{h}\right|$ & erc \\
\hline 11875 & 6.369146 & 6.423856 & 0.054711 & - \\
31969 & 6.394034 & 6.423856 & 0.029823 & 1.84 \\
63693 & 6.404705 & 6.423856 & 0.019151 & 1.92 \\
131470 & 6.412565 & 6.423856 & 0.011291 & 2.19
\end{tabular}

6.2. Eigenvalues of the curl on a rectangular box. For the last test, we have chosen a simple geometry for which, to the best of the authors' knowledge, there is no known analytical solution: a rectangular box. In particular, we have considered the hexahedron $\Omega:=(-0.5,0.5) \times(-0.4,0.4) \times(-0.6,0.6)$. Notice that, as in the previous tests, because of the symmetry of the domain, $\lambda$ is an eigenvalue of Problem 1 is and only if $-\lambda$ is another eigenvalue of the same problem. Therefore each eigenvalue $\lambda^{2}$ of Problem 3 has multiplicity at least two.

We have used several regular meshes as those shown in Figure 3.

On each mesh we have computed the six smallest eigenvalues $\lambda_{h, 1}^{2} \leq \ldots \leq \lambda_{h, 6}^{2}$. In this case, $\lambda_{h, 2 k-1}^{2}$ and $\lambda_{h, 2 k}^{2}$ converge to a same limit $\lambda_{k}^{2}, k=1,2,3$, which is a double eigenvalue of Problem 3.

In absence of an analytical solution, we have estimated the order of convergence by means of a least-squares fitting of the model

$$
\widehat{\lambda}_{h, k} \approx \lambda_{\mathrm{ex}}+C h^{t},
$$



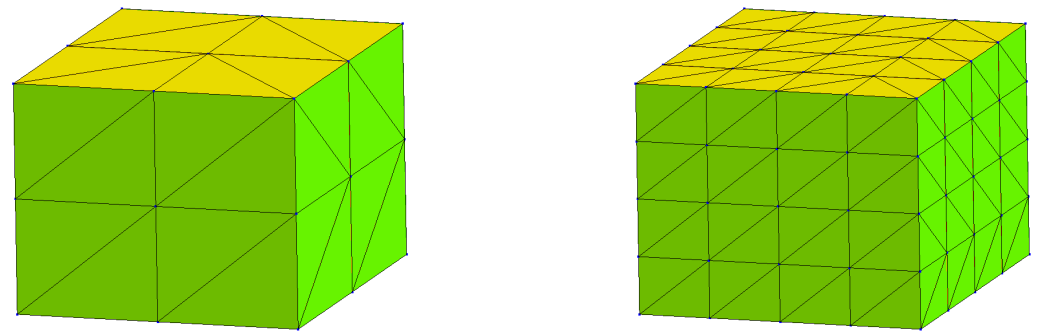

FiguRE 3. Meshes on the rectangular box.

where $\widehat{\lambda}_{h, k}:=\left(\lambda_{h, 2 k-1}+\lambda_{h, 2 k}\right) / 2$.

Table 3 shows the three smallest eigenvalues computed on several meshes. As in the previous examples, $N_{h}$ denotes the corresponding number of tetrahedra. For each eigenvalue, the table also includes the extrapolated more accurate approximation $\lambda_{\text {ex }}$ and the estimated order of convergence $t$ obtained with this fitting. The obtained orders of convergence are again close to $\mathcal{O}\left(h^{2}\right)$, as predicted by the theory.

TABLE 3. Rectangular box. Computed and extrapolated eigenvalues and computed orders of convergence.

\begin{tabular}{c|cccc|c|c} 
& $N_{h}=10368$ & $N_{h}=34992$ & $N_{h}=82944$ & $N_{h}=162000$ & $\lambda_{\text {ex }}$ & order \\
\hline$\widehat{\lambda}_{h, 1}$ & 7.4360 & 7.4337 & 7.4329 & 7.4325 & 7.4319 & 2.02 \\
$\widehat{\lambda}_{h, 2}$ & 7.7666 & 7.7724 & 7.7741 & 7.7751 & 7.7763 & 2.19 \\
$\widehat{\lambda}_{h, 3}$ & 8.0530 & 8.0726 & 8.0802 & 8.0836 & 8.0909 & 1.81
\end{tabular}

To compute the eigenfunctions, we have used Problem 6 as in the previous test. Figure 4 shows the eigenfunction corresponding to the third smallest positive eigenvalue. Those corresponding to the first and the second one are essentially similar to rotations of this. Finally, the eigenfunctions corresponding to the negative eigenvalues are obtained from those of the positive ones by means of a symmetry.

\section{APPENDIX}

In this appendix, we consider a finite element approximation of Problem 2. The simplest minded approach would consist of using the finite element spaces $\mathcal{N}_{h} \subset$ $\mathrm{H}(\mathbf{c u r l} ; \Omega)$ and $\mathcal{L}_{h} \subset \mathrm{H}^{1}(\Omega)$ for a direct discretization of this problem. However, such a procedure leads to a spectral problem for an operator which is not compact and a property analogous to P1 (typical for the spectral approximation of noncompact operators) does not seem to hold, either. To circumvent this drawback, we consider the following problem, which only differs from Problem 2 in that the space $\mathrm{H}(\operatorname{curl} ; \Omega)$ has been substituted by $\mathcal{Z}$.

Problem 5. Find $\lambda \in \mathbb{C}$ and $(\boldsymbol{u}, \varphi) \in \mathcal{Z} \times \mathrm{H}^{1}(\Omega) / \mathbb{C},(\boldsymbol{u}, \varphi) \neq \mathbf{0}$, such that

$$
\begin{aligned}
& \int_{\Omega} \operatorname{curl} \boldsymbol{u} \cdot \operatorname{curl} \overline{\boldsymbol{v}}+\int_{\Omega} \nabla \varphi \cdot \overline{\boldsymbol{v}}=\lambda \int_{\Omega} \boldsymbol{u} \cdot \operatorname{curl} \overline{\boldsymbol{v}} \quad \forall \boldsymbol{v} \in \mathcal{Z}, \\
& \int_{\Omega} \boldsymbol{u} \cdot \nabla \bar{\psi}=0 \quad \forall \psi \in \mathrm{H}^{1}(\Omega) / \mathbb{C} .
\end{aligned}
$$




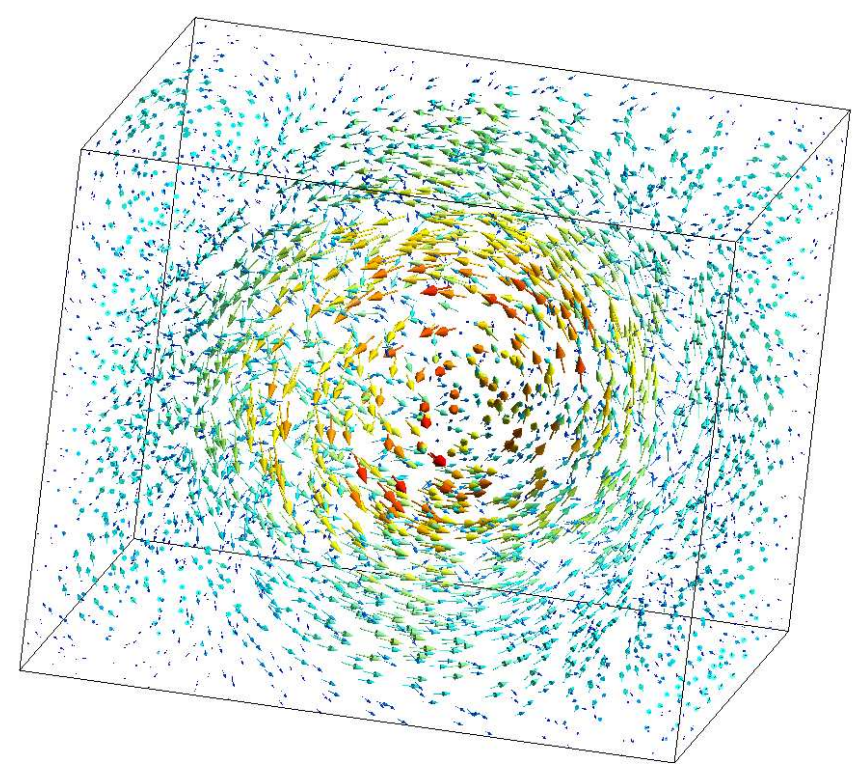

FIgURE 4. Eigenfunction of the curl on a rectangular box.

The following result shows that this is actually equivalent to Problem 2.

Proposition 7.1. Problems 2 and 5 have the same set of solutions.

Proof. Let $(\lambda, \boldsymbol{u}, \varphi)$ be a solution of Problem 2. By virtue of Proposition 3.1, $\operatorname{curl} \boldsymbol{u} \cdot \boldsymbol{n}=\lambda \boldsymbol{u} \cdot \boldsymbol{n}=0$ on $\Gamma$, so that $\boldsymbol{u} \in \mathcal{Z}$. Hence, $(\lambda, \boldsymbol{u}, \varphi)$ solves Problem 5.

Conversely, let $(\lambda, \boldsymbol{u}, \varphi)$ be a solution of Problem 5 . Proceeding as done to prove Proposition 3.1, we obtain $\varphi=0, \boldsymbol{u} \in \mathrm{H}_{0}\left(\operatorname{div}^{0} ; \Omega\right)$, and $\operatorname{curl}(\operatorname{curl} \boldsymbol{u}-\lambda \boldsymbol{u})=\mathbf{0}$ in $\Omega$. Hence, for $\boldsymbol{u} \in \mathcal{Z}$, we have that $\operatorname{curl} \boldsymbol{u}-\lambda \boldsymbol{u} \in \mathrm{H}\left(\operatorname{curl}^{0} ; \Omega\right) \cap \mathrm{H}_{0}\left(\operatorname{div}^{0} ; \Omega\right)=\{\mathbf{0}\}$. Consequently, $\operatorname{curl} \boldsymbol{u}=\lambda \boldsymbol{u}$, and, whence, $(\lambda, \boldsymbol{u}, \varphi)$ solves Problem 2.

Let $\mathcal{Z}_{h} \subset \mathcal{Z}$ and $\mathcal{L}_{h} \subset \mathrm{H}^{1}(\Omega)$ be the finite element spaces defined in Sections 4 and 5 , respectively. We consider the following discretization of Problem 5:

Problem 6. Find $\lambda_{h} \in \mathbb{C}$ and $\left(\boldsymbol{u}_{h}, \varphi_{h}\right) \in \mathcal{Z}_{h} \times \mathcal{L}_{h} / \mathbb{C},\left(\boldsymbol{u}_{h}, \varphi_{h}\right) \neq \mathbf{0}$, such that

$$
\begin{aligned}
& \int_{\Omega} \operatorname{curl} \boldsymbol{u}_{h} \cdot \operatorname{curl} \overline{\boldsymbol{v}}_{h}+\int_{\Omega} \nabla \varphi_{h} \cdot \overline{\boldsymbol{v}}_{h}=\lambda_{h} \int_{\Omega} \boldsymbol{u}_{h} \cdot \operatorname{curl} \overline{\boldsymbol{v}}_{h} \quad \forall \boldsymbol{v}_{h} \in \mathcal{Z}_{h}, \\
& \int_{\Omega} \boldsymbol{u}_{h} \cdot \nabla \bar{\psi}_{h}=0 \quad \forall \psi_{h} \in \mathcal{L}_{h} / \mathbb{C} .
\end{aligned}
$$

Our aim is to prove that the eigenvalues and eigenfunction of Problem 5 are well approximated by those of Problem 6. With this end, we will apply the classical theory for mixed eigenvalue problems of the so-called type Q1 reported in [17, Section 3]. The first step is to show that all the following properties, which correspond to assumptions (3.12)-(3.16) from this reference, are fulfilled in our case: 
- there exists $\alpha_{1}>0$ such that

$$
\int_{\Omega}|\operatorname{curl} \boldsymbol{v}|^{2} \geq \alpha_{1}\|\boldsymbol{v}\|_{\operatorname{curl}, \Omega}^{2} \quad \forall \boldsymbol{v} \in \mathcal{V}
$$

where, we recall, $\mathcal{V}=\left\{\boldsymbol{v} \in \mathcal{Z}: \int_{\Omega} \boldsymbol{v} \cdot \nabla \bar{\psi}=0 \quad \forall \psi \in \mathrm{H}^{1}(\Omega) / \mathbb{C}\right\}$

- there exists $\beta_{1}>0$ such that

$$
\sup _{\boldsymbol{v} \in \mathcal{Z}} \frac{\left|\int_{\Omega} \boldsymbol{v} \cdot \nabla \bar{\psi}\right|}{\|\boldsymbol{v}\|_{\mathbf{c u r l}, \Omega}} \geq \beta_{1}|\psi|_{1, \Omega} \quad \forall \psi \in \mathrm{H}^{1}(\Omega) / \mathbb{C}
$$

- there exists $\alpha_{2}>0$, independent of $h$, such that

$$
\int_{\Omega}\left|\operatorname{curl} \boldsymbol{v}_{h}\right|^{2} \geq \alpha_{2}\left\|\boldsymbol{v}_{h}\right\|_{\text {curl }, \Omega}^{2} \quad \forall \boldsymbol{v}_{h} \in \mathcal{V}_{h}
$$

where, we recall, $\mathcal{V}_{h}=\left\{\boldsymbol{v}_{h} \in \mathcal{Z}_{h}: \int_{\Omega} \boldsymbol{v}_{h} \cdot \nabla \bar{\psi}_{h}=0 \quad \forall \psi_{h} \in \mathcal{L}_{h} / \mathbb{C}\right\}$;

- there exists $\beta_{2}>0$, independent of $h$, such that

$$
\sup _{\boldsymbol{v}_{h} \in \mathcal{Z}_{h}} \frac{\left|\int_{\Omega} \boldsymbol{v}_{h} \cdot \nabla \bar{\psi}_{h}\right|}{\left\|\boldsymbol{v}_{h}\right\|_{\mathbf{c u r l}, \Omega}} \geq \beta_{2}\left|\psi_{h}\right|_{1, \Omega} \quad \forall \psi_{h} \in \mathcal{L}_{h} / \mathbb{C}
$$

- for each $(\boldsymbol{v}, \psi) \in \mathcal{Z} \times \mathrm{H}^{1}(\Omega) / \mathbb{C}$,

$$
\lim _{h \rightarrow 0} \inf _{\left(\boldsymbol{v}_{h}, \psi_{h}\right) \in \mathcal{Z}_{h} \times \mathcal{L}_{h} / \mathbb{C}}\left(\left\|\boldsymbol{v}-\boldsymbol{v}_{h}\right\|_{\mathbf{c u r l}, \Omega}+\left|\psi-\psi_{h}\right|_{1, \Omega}\right)=0 .
$$

The ellipticity in the kernels (7.1) and (7.3) follow from [1, Corollary 3.16] and [1, Proposition 4.6], respectively. The inf-sup conditions (7.2) and (7.4) are easily checked by taking $\boldsymbol{v}=\nabla \psi$ and $\boldsymbol{v}_{h}=\nabla \psi_{h}$, respectively. Finally, the density result (7.5) follows immediately from Proposition 3.4, the fact that for a smooth $\boldsymbol{v} \in \mathcal{Z}$ its Nédélec interpolant satisfies $I_{h}^{N} \boldsymbol{v} \in \mathcal{Z}_{h}$ (cf. the proof of Theorem 4.6), and standard approximation properties of the Nédélec and the Lagrange interpolants.

The solution operator for Problem 5 is defined as follows:

$$
\begin{aligned}
G: \mathcal{Z} \times \mathrm{H}^{1}(\Omega) / \mathbb{C} & \longrightarrow \mathcal{Z} \times \mathrm{H}^{1}(\Omega) / \mathbb{C}, \\
(\boldsymbol{f}, g) & \longmapsto G(\boldsymbol{f}, g):=(\boldsymbol{w}, \xi),
\end{aligned}
$$

with $(\boldsymbol{w}, \xi) \in \mathcal{Z} \times \mathrm{H}^{1}(\Omega) / \mathbb{C}$ such that

$$
\begin{aligned}
& \int_{\Omega} \operatorname{curl} \boldsymbol{w} \cdot \operatorname{curl} \overline{\boldsymbol{v}}+\int_{\Omega} \nabla \xi \cdot \overline{\boldsymbol{v}}=\int_{\Omega} \boldsymbol{f} \cdot \operatorname{curl} \overline{\boldsymbol{v}} \quad \forall \boldsymbol{v} \in \mathcal{Z}, \\
& \int_{\Omega} \boldsymbol{w} \cdot \nabla \bar{\psi}=0 \quad \forall \psi \in \mathrm{H}^{1}(\Omega) / \mathbb{C} .
\end{aligned}
$$

Once more, $\lambda$ is an eigenvalue of Problem 5 if and only if $\mu=\frac{1}{\lambda}$ is a non-zero eigenvalue of $G$, with the same eigenfunctions. An additional assumption needed to apply the approximation results for mixed eigenvalue problems of type Q1 from [17, Section 3] is that $G$ has to be compact. This and other properties of this operator are established in the following lemma.

Lemma 7.2. For all $(\boldsymbol{f}, g) \in \mathcal{Z} \times \mathrm{H}^{1}(\Omega) / \mathbb{C}, G(\boldsymbol{f}, g)=(\boldsymbol{w}, 0)$, with $\boldsymbol{w} \in \mathrm{H}_{0}\left(\operatorname{div}^{0} ; \Omega\right)$. Moreover, $\boldsymbol{w} \in \mathrm{H}^{s}(\mathbf{c u r l} ; \Omega)$ and $G$ is compact.

Proof. Let $(\boldsymbol{f}, g) \in \mathcal{Z} \times \mathrm{H}^{1}(\Omega) / \mathbb{C}$. Proceeding again as done to prove Proposition 3.1, we obtain $G(\boldsymbol{f}, g)=(\boldsymbol{w}, 0)$, with $\boldsymbol{w} \in \mathrm{H}_{0}\left(\operatorname{div}^{0} ; \Omega\right)$ and $\operatorname{curl}(\operatorname{curl} \boldsymbol{w})=$ $\operatorname{curl} \boldsymbol{f}$ in $\Omega$. Therefore, $\boldsymbol{w}, \operatorname{curl} \boldsymbol{w} \in \mathrm{H}(\operatorname{curl} ; \Omega) \cap \mathrm{H}_{0}\left(\operatorname{div}^{0} ; \Omega\right) \hookrightarrow \mathrm{H}^{s}(\Omega)^{3}$. Hence, 
$\boldsymbol{w} \in \mathrm{H}^{s}(\mathbf{c u r l} ; \Omega)$, which is compactly included in $\mathrm{H}(\mathbf{c u r l} ; \Omega)$, and we end the proof.

The spectral approximation theory for mixed problems of type Q1 from [17, Section 3] also involves a formal adjoint operator $G_{*}$. In the present case, this operator is defined for $(\boldsymbol{f}, g) \in \mathcal{Z} \times \mathrm{H}^{1}(\Omega) / \mathbb{C}$ by $G_{*}(\boldsymbol{f}, g):=\left(\boldsymbol{w}_{*}, \xi_{*}\right)$, with $\left(\boldsymbol{w}_{*}, \xi_{*}\right) \in$ $\mathcal{Z} \times \mathrm{H}^{1}(\Omega) / \mathbb{C}$ being the solution of the adjoint problem:

$$
\begin{aligned}
& \int_{\Omega} \operatorname{curl} \boldsymbol{v} \cdot \operatorname{curl} \overline{\boldsymbol{w}}_{*}+\int_{\Omega} \boldsymbol{v} \cdot \nabla \bar{\xi}_{*}=\int_{\Omega} \boldsymbol{v} \cdot \operatorname{curl} \overline{\boldsymbol{f}} \quad \forall \boldsymbol{v} \in \mathcal{Z}, \\
& \int_{\Omega} \nabla \psi \cdot \overline{\boldsymbol{w}}_{*}=0 \quad \forall \psi \in \mathrm{H}^{1}(\Omega) / \mathbb{C} .
\end{aligned}
$$

In general, for each eigenvalue $\mu$ of $G, \bar{\mu}$ is an eigenvalue of $G_{*}$ with the same ascent $\alpha$ and with invariant subspace $\mathcal{E}^{*}:=\operatorname{Ker}\left(\left(\bar{\mu} I-G_{*}\right)^{\alpha}\right)$. In our case, $\mu \in \mathbb{R}$ and, as an immediate consequence of Proposition 3.3, we have that $G_{*}=G$. However, since $G_{*}$ is just a formal adjoint, we cannot claim that $G$ is self-adjoint. Nevertheless, we prove in the following lemma that its eigenvalues are non-defective.

Lemma 7.3. The ascent of any non-zero eigenvalue of $G$ is one.

Proof. By contradiction. Let $(\mu,(\boldsymbol{u}, \varphi))$ be an eigenpair of $G, \mu \neq 0$, and let us assume that $G$ has a corresponding generalized eigenfunction; namely, $G(\boldsymbol{u}, \varphi)=$ $\mu(\boldsymbol{u}, \varphi),(\boldsymbol{u}, \varphi) \neq \mathbf{0}$, and there exists $(\widetilde{\boldsymbol{u}}, \widetilde{\varphi})$ such that $G(\widetilde{\boldsymbol{u}}, \widetilde{\varphi})=\mu(\widetilde{\boldsymbol{u}}, \widetilde{\varphi})+(\boldsymbol{u}, \varphi)$. Hence, $\varphi=\widetilde{\varphi}=0$ and, by using the definition of $G$ for $\boldsymbol{f}=\boldsymbol{u}$ with test function $\boldsymbol{v}=\widetilde{\boldsymbol{u}}$ and for $\boldsymbol{f}=\widetilde{\boldsymbol{u}}$ with test function $\boldsymbol{v}=\boldsymbol{u}$, we respectively obtain

$$
\begin{aligned}
& \mu \int_{\Omega} \operatorname{curl} \boldsymbol{u} \cdot \operatorname{curl} \overline{\widetilde{\boldsymbol{u}}}=\int_{\Omega} \boldsymbol{u} \cdot \operatorname{curl} \overline{\widetilde{\boldsymbol{u}}} \\
& \mu \int_{\Omega} \operatorname{curl} \widetilde{\boldsymbol{u}} \cdot \operatorname{curl} \overline{\boldsymbol{u}}+\int_{\Omega} \operatorname{curl} \boldsymbol{u} \cdot \operatorname{curl} \overline{\boldsymbol{u}}=\int_{\Omega} \widetilde{\boldsymbol{u}} \cdot \operatorname{curl} \overline{\boldsymbol{u}}
\end{aligned}
$$

Subtracting the conjugate of the first equation from the second one and using that $\mu \in \mathbb{R}$, we have that

$$
\mu \int_{\Omega}|\operatorname{curl} \boldsymbol{u}|^{2}=\int_{\Omega}(\widetilde{\boldsymbol{u}} \cdot \operatorname{curl} \overline{\boldsymbol{u}}-\overline{\boldsymbol{u}} \cdot \operatorname{curl} \tilde{\boldsymbol{u}})=0,
$$

the last equality because of Proposition 3.3. Therefore, for $\mu \neq 0$, by virtue of (7.1), $\boldsymbol{u}=\mathbf{0}$. Since $\varphi=0$, too, this leads to a contradiction and we end the proof.

Now, we are in a position to write the following convergence result, which is a direct consequence of [17, Theorem 3.1].

Theorem 7.4. Let $\lambda$ be an eigenvalue of Problem 5 of finite-multiplicity $m$. Let $\mathcal{E} \subset \mathcal{Z} \times \mathrm{H}^{1}(\Omega) / \mathbb{C}$ be the corresponding eigenspace. Then, there exist exactly $m$ eigenvalues $\lambda_{h}^{(1)}, \ldots, \lambda_{h}^{(m)}$ of Problem 6 (repeated accordingly to their respective multiplicities) which converge to $\lambda$ as $h$ goes to zero.

Let $\mathcal{E}_{h}$ be the direct sum of the eigenspaces corresponding to $\lambda_{h}^{(1)}, \ldots, \lambda_{h}^{(m)}$ and $\widehat{\gamma}_{h}:=\delta\left(\mathcal{E}, \mathcal{Z}_{h} \times \mathcal{L}_{h} / \mathbb{C}\right)$. Then,

$$
\widehat{\delta}\left(\mathcal{E}, \mathcal{E}_{h}\right) \leq C \widehat{\gamma}_{h}
$$

and

$$
\left|\lambda-\lambda_{h}^{(i)}\right| \leq C \widehat{\gamma}_{h}^{2}, \quad i=1, \ldots, m
$$


Lemma 7.2 and the same arguments used in the proof of Theorem 4.6 allow us to show that there exists $C>0$, independent of $h$, such that $\widehat{\gamma}_{h} \leq C h^{r_{k}}$, with $r_{k}$ as defined in (4.1). This, together with Theorem 7.4, imply that the eigenvalues and eigenfunctions of Problem 6 converge to those of Problem 5 with an optimal order.

The matrix form of Problem 6 is a generalized eigenvalue problem which involves two non-definite matrices. However, in spite of this, it is well-posed. In fact, it is easy to check that $\left(\lambda_{h}, \boldsymbol{u}_{h}, \varphi_{h}\right)$ is a solution of Problem 6 if and only if $\varphi_{h}=0$ and $\left(\lambda_{h}, \boldsymbol{u}_{h}\right)$ is a solution of the following one: find $\lambda_{h} \in \mathbb{C}$ and $\boldsymbol{u}_{h} \in \mathcal{V}_{h}, \boldsymbol{u}_{h} \neq \mathbf{0}$, such that

$$
\int_{\Omega} \operatorname{curl} \boldsymbol{u}_{h} \cdot \operatorname{curl} \overline{\boldsymbol{v}}_{h}=\lambda_{h} \int_{\Omega} \boldsymbol{u}_{h} \cdot \operatorname{curl} \overline{\boldsymbol{v}}_{h} \quad \forall \boldsymbol{v}_{h} \in \mathcal{V}_{h} .
$$

The matrix form of the above problem involves two Hermitian matrices, that of the right hand side being so because of Proposition 3.3. Moreover, the matrix on the left hand side is positive definite because of (7.3). Therefore, the eigenvalues of this discrete problem are real and non-defective.

Problem 6 is equivalent to the well-posed generalized eigenvalue problem (7.6). However, since there is no basis available for $\mathcal{V}_{h}$, its computer implementation requires dealing with Problem 6, in spite of its degeneracy, rather than with (7.6).

\section{ACKNOWLEDGMENT}

Authors thank Alberto Maltz for bringing this problem to their attention.

\section{REFERENCES}

[1] C. Amrouche, C. Bernardi, M. Dauge, and V. Girault, Vector potentials in three-dimensional non-smooth domains, Math. Methods Appl. Sci., 21 (1998) 823-864.

[2] E. Beltrami, Considerazioni idrodinamiche, Rend. Inst. Lombardo Acad. Sci. Let., 22 (1889) 122-131. (English translation: Considerations on hydrodynamics, Int. J. Fusion Energy, 3 (1985) 53-57.)

[3] A. Bermúdez, R. Rodríguez, and P. Salgado, Numerical analysis of electric field formulations of the eddy current model, Numer. Math., 102 (2005) 181-201.

[4] D. Boffi, Finite element approximation of eigenvalue problems, Acta Numer., 19 (2010) 1120.

[5] T.Z. Boulmezaud and T. Amari, Approximation of linear force-free fields in bounded 3-D domains, Math. Comp. Model., 31 (2000) 109-129.

[6] _ A finite-element method for computing nonlinear force-free fields, Math. Comput. Model., 34 (2001) 903-920.

[7] T.Z. Boulmezaud, Y. Maday, and T. Amari, On the linear force-free fields in bounded and unbounded three-dimensional domains, $\mathrm{M}^{2}$ AN Math. Model. Numer. Anal., 33 (1999) 359393.

[8] A. Buffa, M. Costabel, and D. Sheen, On traces for $\mathrm{H}(\mathbf{c u r l} ; \Omega)$ in Lipschitz domains, J. Math. Anal. Appl., 276 (2002) 845-867.

[9] J. Cantarella, D. De Turck, H. Gluck, and M. Teytel, The spectrum of the curl operator on spherically symmetric domains, Phys. Plasmas, 7 (2000) 2766-2775.

[10] S. Chandrasekhar and L. Woltjer, On force-free magnetic fields, Proc. Nat. Acad. Sci. USA, 44 (1958) 842-847.

[11] S. Chandrasekhar and P.C. Kendall, On force-free magnetic fields, Astrophys. J., 126 (1957) 457-460.

[12] J. Descloux, N. Nassif, and J. Rappaz, On spectral approximation. I. The problem of convergence, RAIRO Anal. Numér., 12 (1978) 97-112.

[13] _ On spectral approximation. II. Error estimates for the Galerkin method, RAIRO Anal. Numér., 12 (1978) 113-119.

[14] V. Girault and P.-A Raviart, Finite Element Approximations of the Navier-Stokes Equations, Theory and Algorithms. Springer, Berlin, 1986. 
[15] A. Lakhtakia, Victor Trkal, Beltrami fields and Trkalian flows, Czech J. Phys., 44 (1994) 89-96.

[16] S. Meddahi and V. Selgas, A mixed-FEM and BEM coupling for a three-dimensional eddy current problem, M² AN Math. Model. Numer. Anal., 37 (2003) 291-318.

[17] B. Mercier, J. Osborn, J. Rappaz, and P.-A. Raviart, Eigenvalue approximation by mixed and hybrid methods, Math. Comp., 36 (1981) 427-453.

[18] P. Monk, Finite Element Methods for Maxwell's Equations. Oxford University Press, New York, 2003.

[19] E.C. Morse, Eigenfunctions of the curl in cylindrical geometry, J. Math. Phys., 46 (2005) 113511, 13pp.

[20] J.C. Nédélec, Mixed finite elements in $\mathbb{R}^{3}$, Numer. Math., 35 (1980) 315-341.

[21] J.B. Taylor, Relaxation of toroidal and generation of reverse magnetic fields, 33 (1974) 11391141.

[22] V. Trkal, Poznámka k hydrodynamice vazkých tekutin, Časopis pro Pĕstování Mathematiky a Fysiky, 48 (1919) 302-311. (English translation: A note on the hydrodynamics of viscous fluids, Czech J. Phys., 44 (1994) 97-106.)

[23] L. Woltjer, A theorem on force-free magnetic fields, Proc. Nat. Acad. Sci. USA, 44 (1958) 489-491.

[24] _ The crab nebula, Bull. Astron. Inst. Neth., 14 (1958) 39-80.

[25] Z. Yoshida and Y. Giga, Remarks on spectra of operator rot, Math. Z., 204 (1990) 235-245.

$\mathrm{CI}^{2} \mathrm{MA}$, Departamento de Ingeniería Matemática, Universidad de Concepción, Casilla 160-C, Concepción, Chile

E-mail address: rodolfo@ing-mat.udec.cl

CI $^{2}$ Ma, Departamento de Ingeniería Matemática, Universidad de Concepción, Casilla 160-C, Concepción, Chile

E-mail address: pvenegas@ing-mat.udec.cl 